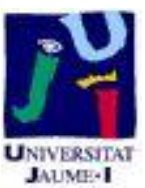

Título artículo / Títol article: Process-oriented tolerancing using the extended stream of variation model

Autores / Autors

Abellán Nebot, José Vicente ; Liu, Jian ; Romero

Subirón, Fernando

Revista:

Computers in Industry, 2013, vol. 64, no 5

Versión / Versió:

Preprint del autor

Cita bibliográfica / Cita bibliogràfica (ISO 690):

url Repositori UJI:
ABELLÁN-NEBOT, José V.; LIU, Jian; SUBIRÓN, F. Romero. Process-oriented tolerancing using the extended stream of variation model. Computers in Industry, 2013, vol. 64, no 5, p. 485-498

http://hdl.handle.net/10234/88409 


\title{
Process-Oriented Tolerancing using the Extended Stream of Variation Model
}

\author{
José V. Abellan-Nebot ${ }^{\mathrm{a}, *}$, Jian Liu $^{\mathrm{b}}$, F. Romero Subirón ${ }^{\mathrm{a}}$ \\ ${ }^{a}$ Department of Industrial Engineering and Design, Universitat Jaume I \\ Av. Sos Baynat s/n, Castellon 12071, Spain \\ ${ }^{b}$ Department of Systems and Industrial Engineering, University of Arizona \\ Tucson, AZ 85718, United States
}

\begin{abstract}
Current works on process-oriented tolerancing for multi-station manufacturing processes (MMPs) have been mainly focused on allocating fixture tolerances to ensure part quality specifications at a minimum manufacturing cost. Some works have also included fixture maintenance policies into the tolerance allocation problem since they are related to both manufacturing cost and final part quality. However, there is a lack of incorporation of other factors that lead to increase of manufacturing cost and degrade of product quality, such as cutting-tool wear and machine-tool thermal state. The allocation of the admissible values of these process variables may be critical due to their impact on cutting-tool replacement and quality loss costs. In this paper, the process-oriented tolerancing is expanded based on the recently developed, extended stream of variation (SoV) model, which explicitly represents the influence of machining process variables in the variation propagation along MMPs. In addition, the probability distribution functions (pdf) for some machining process variables are analyzed, and a procedure to derive part quality constraints according to GD\&T specifications is also shown. With this modeling capability extension, a complete process-oriented tolerancing can be conducted, reaching a real minimum manufacturing cost. In order to demonstrate the advantage of the proposed methodology over a conventional method, a case study is analyzed in detail.
\end{abstract}

Keywords: stream-of-variation, variation propagation, process-oriented tolerancing, machining-induced variations, tool replacement, fixture maintenance

\section{Introduction}

Machining operations are inherently imperfect in fabricating parts. This is due to the numerous process variables that affect the final quality of products, such as machine-tool thermal expansion, cutting-tool wear, fixture error, etc. The inherent variability of machining processes requires to specify dimensional and geometrical tolerances on raw and machined surfaces to ensure final product functionality. Product tolerancing defines the variability allowed for each key variable that characterizes the functional requirements of the product, named the key product characteristics (KPCs). There are two basic directions in tolerancing research: (a) tolerance analysis and (b) tolerance synthesis [1]. Tolerance analysis predicts the variation of the final product given the tolerance of each part using

\footnotetext{
${ }^{*}$ Corresponding author. Tel.: +34 964 728186; fax:+34 964728170 .

Email addresses: abellan@uji.es (José V. Abellan-Nebot), jianliu@sie.arizona.edu (Jian Liu), fromero@uji.es (F. Romero Subirón)
} 
a mathematical model of tolerance accumulation such as the worst case model or the root square sum [2, Chapter 9]. Tolerance synthesis, or tolerancing, focuses on assigning tolerance specifications to individual manufacturing features on a part to ensure product functionality and minimize manufacturing cost. In the literature, the traditional tolerancing approach is product-oriented. It mainly focuses on assigning tolerance to product variables, such as dimensions of final product and parts. However, this approach only considers limited a priori knowledge about manufacturing capabilities and manufacturing costs of specific operations, and does not explicitly specify the allowable variability of the process variables, such as those related to tooling variations due to wear, thermal distortions or manufacturing accuracy. Recently, the process-oriented tolerancing approach was proposed by [3]. This approach is essentially a tolerance transfer method where the quality specification of the final product is ensured by optimally assigning tolerances of process variables throughout the manufacturing process.

In a multi-station manufacturing process (MMP), the process variables, also referred as key control characteristics (KCCs), are the root causes of the process faults that negatively impact on the KPCs. These KCCs define the working condition of the tools (machine-tools, fixtures and cuttingtools) that are used to fabricate a part. In the process-oriented tolerancing approach, the incorporation of KCCs into tolerance models leads to the integration of tolerancing with process maintenance and operation strategies. As a result, a more comprehensive function cost can be considered to find out the optimal tolerance allocation that minimizes the total manufacturing cost. The main challenge of process-oriented tolerancing is the definition of a mathematical model that describes the effect of KCC variations on the KPCs in a station of a MMP. Such effects on KPC variations may be propagated to downstream stations and accumulated to the final product. Recently, this type of variation propagation in MMPs has been successfully modeled by applying the stream of variation (SoV) modeling [4]. The SoV modeling is a systematic methodology to derive the KPC-KCC relationship based on engineering domain knowledge on the product/process design [5]. Based on SoV models, many quality improvement activities have been conducted on MMPs, such as process diagnosis [6], sensor placement for in-process inspection [7, 8], quality prediction [9-11] and dimensional quality control $[12,13]$. However, only few works have been focused on process-oriented tolerancing [3, 1416]. In this specific field, Ding et al. [3] applied the process-oriented tolerancing approach to allocate product and process tolerances in a multi-station assembly process (MAP). The KCCs modeled were the variability of fixture locators caused by their degradation. Considering reciprocal functions as cost-tolerance functions, the optimal tolerance of KCCs with the minimum manufacturing cost was allocated by solving a constrained optimization problem. Similar problem was described by Chen et al. [14], who expanded Ding's work to integrate the process-oriented tolerancing with the fixture maintenance planning. Tool fabrication cost, fixture maintenance cost and quality loss functions were considered together to optimize the process tolerance allocation and the frequency of fixture maintenance operations in MAPs. The main goal of this work was to present an integrated method to analyze maintenance operations and process design together with the resulting assembly quality. These two works established the fundamentals of process-oriented tolerancing through the use of the SoV model. However, their works were focused on MAPs, where only fixture-induced variations are of interest for tolerance allocating purposes.

Process-oriented tolerancing has been less explored on machining systems, where unlike MAPs, a large number of process variables with different cost functions should be considered. In this field, Huang et al. [15] developed a tolerance allocation methodology considering as process variables the 
deviations of fixture locators and the generic deviation of the cutting-tool movements of the machinetool at each station. This tolerance allocation problem seeks to maximize the variance of these process variables constrained to part quality specifications, assuming that all process variables are independent to each other and follow a normal distribution. Recently, Liu et al. [16] studied the use of the SoV model to determine optimal setup planning that ensures product quality with minimum cost, assuming that cost is inversely proportional to the necessary process precision. In their work, both fixture-induced and machining-induced deviations (considering the later as a generic cutting-tool path deviation) were treated as random process deviations.

Four main limitations can be identified in the previous research works. (i) The process variables considered in the machining systems are not comprehensive, and the tolerance allocation is conducted considering locator tolerances and generic cutting-tool path deviations. However, machinetools present other process variables that influence on the cutting-tool path accuracy such as cuttingtool wear, thermal state of the spindle, etc [17]. In fact, a recent research work [18] demonstrated that without considering these process variables in the SoV model, part quality prediction at the end of a MMP may result in important misleading conclusions. Therefore, a complete tolerance allocation requires the inclusion of additional process variables. (ii) The cost to be considered in the tolerance allocation problem should include not only fixture cost (both design and maintenance cost) but also other cost related to machining such as cutting-tool costs, thermal-control costs, etc. (iii) The process variables considered have been assumed to follow a normal distribution $[15,16]$ for tolerance allocation purposes. However, other distributions closer to the real production system should be considered for process variables such as locators wear or cutting-tool wear. (iv) The part quality constraints should be considered as geometric dimensional and tolerancing (GD\&T) specifications, instead of vectorial dimensioning and tolerancing (VD\&T) specifications which are not used in industry, although they can be easily applied by using the SoV model.

In order to illustrate the limitations of current process-oriented tolerancing due to the neglection of specific machining-induced variations, an example of a two-station machining process shown in Fig. 1 is considered. At the first station, the dimension of the machined feature $D_{2}$ is deviated from its nominal value due to the locator tolerance, denoted by $\pm T_{2}$, and the machining-induced variations due to the cutting-tool wear, denoted by $\pm T_{3}$, and those induced by the spindle thermal expansion, denoted by $\pm T_{4}$. Thus, considering the worst case deviation, the tolerance of $D_{2}$ is defined by $T_{1}=T_{2}+T_{3}+T_{4}$. The workpiece is then set up at station 2 , where the dimension of the feature to be machined, $D_{3}$, will be deviated from nominal values in a similar way and thus, its final tolerance will be defined by $T_{5}=T_{6}+T_{7}+T_{8}$. As a result, the KPC of this part defined by the dimension of the feature $D_{4}$ will depend on all previous fixture and machining-induced variations. Its tolerance will be defined as $T_{10}=T_{1}+T_{5}+T_{9}=T_{2}+T_{3}+T_{4}+T_{6}+T_{7}+T_{8}+T_{9}$, which means that if machining-induced variations are not negligible with respect to fixture-induced variations, the achievable tolerance of this KPC depends on the variability of six different KCCs defined by the tolerances $\left\{T_{2}, T_{3}, T_{4}, T_{6}, T_{7}, T_{8}\right\}$. Note that the tolerance $T_{9}$ refers to the tolerance of the dimension $D_{1}$ (raw material) so it is not related to a fixture or machining-induced variation. Neglecting the machining-induced variations will result in allocating a higher tolerance value for fixture locators. However, if in reality machining-induced variations are not negligible, the locator tolerances allocated will generate parts outside specifications since additional sources of variation are present. As a conclusion, a reliable process-oriented tolerancing requires the incorporation of all those KCCs related to machining-induced variations that directly influence on part quality. 


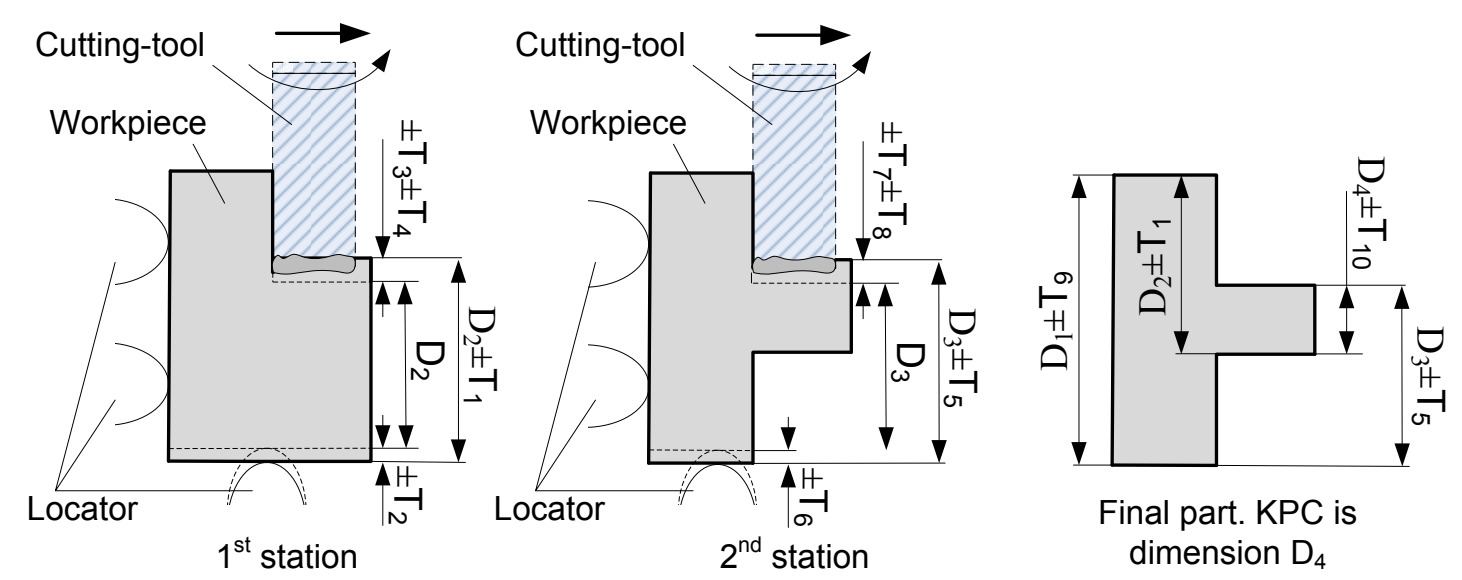

Figure 1: Example of the influence of machining-induced variations on the tolerance allocation problem.

In this paper, a process-oriented tolerancing methodology is proposed to include critical process variables and cost functions, which are not considered yet in existing tolerance allocation approaches. The proposed methodology provides a generic framework for the inclusion of a variety of process variation sources. And specifically, the inclusion of process variables related to thermal errors and cutting-tool wear-induced errors together with their corresponding cost functions are discussed in detail. Furthermore, the probability density function (pdf) of some of the process variables are derived mathematically according to the nature of the machining process. To ensure that the machined part quality is within GD\&T specifications (e.g. position tolerance specification), a methodology to derive the optimization constraints is also presented.

The rest of this paper is organized as follows. Section 2 introduces the formulation of the state space model and the deviation representation mechanism adopted in this paper. Section 3 formulates the process-oriented tolerance allocation problem with the costs associated to fixture maintenance policies and cutting-tool replacement policies. A case study is shown in Section 4 to demonstrate the effectiveness of the proposed process-oriented tolerancing approach. Finally, Section 5 presents the conclusions of the paper.

\section{Stream of Variation modeling for MMPs}

Process-oriented tolerancing requires a variation propagation model to relate the KPCs with the KCCs in a MMP. Various propagation models have been developed according to the nominal product and process design information and off-line analysis of quality-process interactions and inter-station correlations. Mantripragada et al. [19] proposed the datum flow chain concept for MAPs design. For modeling the 2D variation propagation in sheet metal MAPs, Jin et al. [20] developed the state space modeling technique, which was further extended to 3D general assembly processes [16, 21]. For modeling multi-station machining processes, research works [15] and [22] investigated the 3D variation propagation with an approximately linearized state space model. Zhou et al. [9] further generalized the deviation representation and proposed a generic linear state space model based on differential motion vectors (DMVs). A similar model was proposed by Wan et al. [23] including error sources due to workpiece-fixture local contact compliance. Recently, Abellan-Nebot et al. [11] extended the state space model developed in [9] by including specific machining-induced variations 
such as those due to geometric-thermal variations of machine-tools, cutting-tool deflections or cuttingtool wear. In this section, the representation and derivation of the SoV model are briefly introduced.

\subsection{Random deviation representation}

Vectorial dimensioning and tolerancing (VD\&T) was presented as an alternative to represent workpiece tolerances in the early nineties [24]. For variation propagation modeling in MMP, Zhou et al. [9] adopted the DMV approach originated from VD\&T and robotics manipulations in order to model variation feature representation. The DMV approach uses the vectorial representation as in VD\&T except that the orientation vector is based on the three Euler rotating angles instead of using a unit direction vector. To understand the DMV representation, let us consider an ideal workpiece surface plane defined by its local coordinate system $\left(L C S_{1}\right)$. The $L C S_{1}$ can be located by a location vector, $\mathbf{t}_{1}^{R}=\left[t_{1 x}^{R}, t_{1 y}^{R}, t_{1 z}^{R}\right]^{T}$, and an orientation vector, $\boldsymbol{\omega}_{1}^{R}=\left[\omega_{1 \alpha}^{R}, \omega_{1 \beta}^{R}, \omega_{1 \gamma}^{R}\right]^{T}$. Both vectors are defined with respect to (w.r.t.) the reference coordinate system (RCS), denoted as "R". Thus, the $L C S_{1}$ is located by the stacked vector $\left[\left(\mathbf{t}_{1}^{R}\right)^{T},\left(\boldsymbol{\omega}_{1}^{R}\right)^{T}\right]^{T}$. If the surface plane deviates from its nominal location or orientation due to certain errors in the machining process, the actual $L C S_{1}$ deviates by a location $\operatorname{DMV} \mathbf{d}_{1}^{R}=[\Delta x, \Delta y, \Delta z]^{T}$ and an orientation DMV $\mathbf{s}_{1}^{R}=[\Delta \alpha, \Delta \beta, \Delta \gamma]^{T}$. Therefore, the variation feature representation can be expressed by the stacked DMV $\mathbf{x}_{1}^{R}=[\Delta x, \Delta y, \Delta z, \Delta \alpha, \Delta \beta, \Delta \gamma]^{T}$. In this paper, the DMV approach based on the ZYZ Euler rotation order is applied for feature variation representation.

\subsection{SoV model derivation}

In a MMP, the product variation at a certain station consists of two components: the variation induced within a station (fixture and machining-induced variations) and the variation propagated from upstream stations [4]. The second component exists when the features produced by upstream stations are used as datum features to locate the part in the current station. In these cases, the variation from the upstream stations will be accumulated onto current station. Based on the vectorial deviation representation with DMVs, the variation propagation can be described with a state space model as follows. For a MMP with $N$ stations as shown in Fig. 2, the deviations that have been generated on a workpiece after station $k$ are denoted as a state vector, $\mathbf{x}_{k}, k=1,2, \ldots, N$. $\mathbf{x}_{k}$ is a stack of the DMVs of the features defined in the product design. Denoting the variation introduced from station $k-1$ as $\mathbf{x}_{k-1}$, the fixture and machining-induced variations at station $k$ as $\mathbf{u}_{k}$, and the deviations of the $M$ KPCs measured at station $k$ as $\mathbf{y}_{k}$, the variation propagation is formulated as:

$$
\begin{aligned}
& \mathbf{x}_{k}=\mathbf{A}_{k-1} \cdot \mathbf{x}_{k-1}+\mathbf{B}_{k} \cdot \mathbf{u}_{k}+\mathbf{w}_{k}, \\
& \mathbf{y}_{k}=\mathbf{C}_{k} \cdot \mathbf{x}_{k}+\mathbf{v}_{k},
\end{aligned}
$$

where $\mathbf{A}_{k-1} \cdot \mathbf{x}_{k-1}$ represents the variations transmitted through datum features from upstream stations; $\mathbf{B}_{k} \cdot \mathbf{u}_{k}$ represents the variations introduced at station $k$, including both fixture- and machining-induced variations; $\mathbf{C}_{k} \cdot \mathbf{x}_{k}$ represents the measurements of KPC variations, which are defined as a linear combination of manufacturing feature deviations; $\mathbf{w}_{k}$ and $\mathbf{v}_{k}$ are the un-modeled system terms and measurement noise, respectively. Note that a different "station" is considered when a workpiece is removed from its fixture in order to be mounted on another fixture or in the same fixture but with different datum surfaces.

Zhou et al. [9] proposed a generic method to derive the matrices $\mathbf{A}_{k-1}, \mathbf{B}_{k}$, and $\mathbf{C}_{k}$, under the assumptions that the machined workpiece behaves as a rigid body and the magnitudes of random deviations are small enough to ensure the validity of the linearization. However, their model formulation considers only fixture variations and generic cutting-tool path deviations in the term $\mathbf{u}_{k}$, 


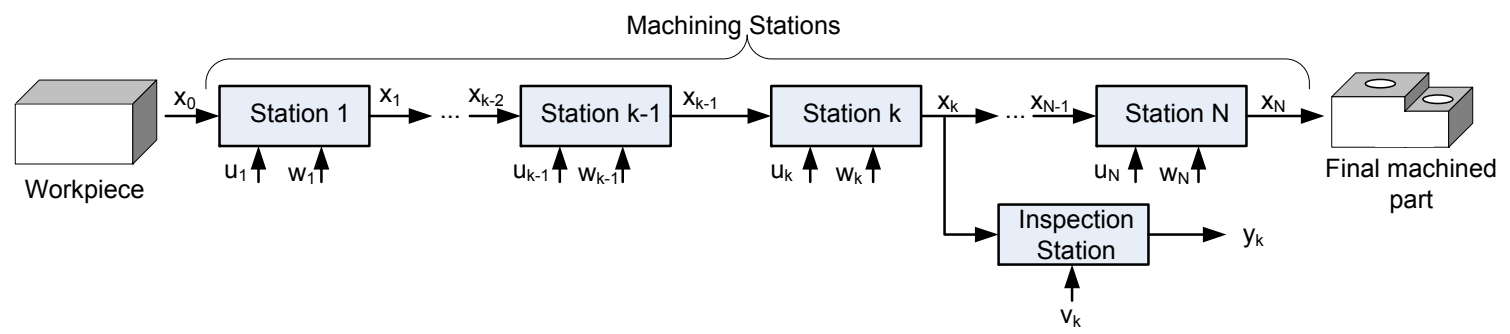

Figure 2: MMP composed of N stations

without explicitly modeling specific machining-induced variations. Abellan-Nebot et al. [11] extended this formulation by including machining machining-induced variations, such as geometricthermal variations, cutting-tool deflections and cutting-tool wear. Without loss of generality, two machining-induced variations are considered in the variation propagation model in this paper: i) machining-induced variations due to cutting-tool wear and ii) machining-induced variations due to spindle thermal expansion. Focusing the research work on small-medium workpiece size with the use of cutter insert plates, these two machining-induced variations can be considered as the most critical KCCs for tolerancing rather than other variation sources, such as cutting-tool deflections or geometric deviations of machine-tool axes [11]. According to the extended SoV model, $\mathbf{B}_{k}$ and $\mathbf{u}_{k}$ terms are expanded in the form of $\mathbf{B}_{k}=\left[\begin{array}{lll}\mathbf{B}_{k}^{f} & \mathbf{B}_{k}^{s} & \mathbf{B}_{k}^{w}\end{array}\right]$ and $\mathbf{u}_{k}=\left[\begin{array}{lll}\mathbf{u}_{k}^{f} & \Delta T_{k}^{s} & V_{k}\end{array}\right]^{T}$. By this state space model formulation, the KPC variations can be related to that of the KCCs, including the fixture locator variations $\left(\mathbf{u}_{k}^{f}\right)$, the spindle thermal expansion $\left(\Delta T_{k}^{s}\right)$ and the cutting-tool wear state $\left(V_{k}\right)$. The matrices $\mathbf{B}_{k}^{f}, \mathbf{B}_{k}^{s}$ and $\mathbf{B}_{k}^{w}$ can be derived following [9] and [11].

\section{Process-oriented tolerance allocation}

\subsection{Assumptions}

In this paper, the following assumptions are made to facilitate the model formulation and derivation:

- Machined workpiece behaves as a rigid body and the magnitudes of all random deviations are small.

- The sources of process variations are assumed to be independent of each other.

- Variability of process variables may be not normally distributed due to the nature of the machining process.

- There are no dominant process variables that impact on the KPC deviations and thus, the variance of KPCs due to the variance of any process variable is very small in comparison to the total variance of the KPCs due to the rest of process variables.

- The manufacturing process is assumed to be centered and thus, it is assumed that there is no mean-shift of KPCs.

- In a fixture device when the maximum admissible wear of a locator is reached, a fixture maintenance action is carried out to replace and calibrate all fixture locators. 


\subsection{Problem Formulation}

For tolerance allocation purposes, we reformulate the matrix $\mathbf{B}_{k}$ as $\left[\begin{array}{llll}\mathbf{B}_{k}^{f} & \mathbf{B}_{k}^{f} & \mathbf{B}_{k}^{s} & \mathbf{B}_{k}^{w}\end{array}\right]$ and the vector $\mathbf{u}_{k}$ as $\left[\left(\mathbf{u}_{k}^{f i}\right)^{T},\left(\mathbf{u}_{k}^{f w}\right)^{T}, \Delta T_{k}^{s}, V_{k}\right]^{T}$ in order to separate the fixture deviation in two independent components: the component due to the accuracy of each locator after mounting and calibrating the fixture device (defined by the term $\mathbf{u}_{k}^{f i}$ ) and the component due to the degradation of each fixture locator during normal operation (defined by the term $\mathbf{u}_{k}^{f w}$ ). If the machined part is inspected at the end of the machining process, Eqs. (1) and (2) can be rewritten in an input-output form as:

$$
\mathbf{Y}=\boldsymbol{\Gamma} \cdot \mathbf{U}+\varepsilon
$$

where $\mathbf{U}=\left[\left(\mathbf{u}_{1}\right)^{T}, \ldots,\left(\mathbf{u}_{N}\right)^{T}\right]^{T}$ is the vector of the KCCs of all stations, and $\mathbf{Y}=\left[y_{1}, \ldots, y_{M}\right]^{T}$ is the vector of the $M$ KPC measurements collected at the end of a MMP. $\Gamma$ and $\varepsilon$ can be obtained as shown in [4].

The process-oriented tolerancing consists of defining the tolerances for all KCCs that minimize the total manufacturing cost and ensuring part quality specifications. Thus, the optimal process-oriented tolerance allocation can be formulated as the following optimization problem:

$$
\min _{\mathbf{T}_{\mathbf{U}}}\left\{C_{T}\left(\mathbf{T}_{\mathbf{U}}\right)\right\}, \quad \text { subjected to } \Psi,
$$

where $C_{T}$ is the total manufacturing cost which is a function of $\mathbf{T}_{\mathbf{U}} ; \mathbf{T}_{\mathbf{U}}=\left[T_{1}, T_{2}, \ldots, T_{\eta}\right]^{T}$ is a vector where the element $T_{\chi}$ represents the tolerance of the $\chi$ th KCC defined in $\mathbf{U}$; and $\boldsymbol{\Psi}$ is a vector of constraints. Considering two types of machining-induced variations (due to tool wear and spindle thermal expansion) and the fixture-induced variations, this optimization problem is to minimize the total manufacturing cost for allocating: i) the initial precision of fixture locators, ii) the admissible maximum wear of fixture locators before a maintenance operation is carried out, and iii) the admissible maximum wear of cutting-tools before a cutting-tool replacement is conducted. The tolerance allocation problem is thus composed of the following cost functions: i) fixture precision cost, ii) fixture maintenance cost, iii) cutting-tool replacement cost, and iv) quality loss cost, which is incurred by the scrap and rework of the parts that fail to meet the quality specification. Furthermore, the vector of constraints is composed of: i) constraints of the non-maintainable KCCs, ii) constraints of the maintainable KCCs, and iii) constraints related to part quality specifications. The following subsections will describe the manufacturing cost functions and the constraints in detail.

\subsection{Definition of manufacturing cost functions}

\subsubsection{Fixture precision cost}

The first group of manufacturing costs is related to the precision of the fixtures to be used. Tighter tolerances of fixturing elements result in higher tooling fabrication and assembly costs. Several algebraic functions have been proposed to calculate fixture precision costs, such as reciprocal, reciprocal power, negative exponential and reciprocal squared functions [2]. For tolerance allocation, the reciprocal function and negative exponential function are widely accepted [25]. In this paper, we assume a fixture layout at each station based on the common 3-2-1 locating principle applied in machining processes [26]. For this fixture layout, the cost function for the $j$ th fixture component (locator) installed at station $k$ is chosen to be a reciprocal function as $[14,16]$ 


$$
C_{j, k}^{L}=\frac{w_{j, k}^{D}}{\Delta l_{j, k}^{D}}, \quad k=1, \ldots, N, \quad j=1, \ldots, 6,
$$

where $\Delta l_{j, k}^{D}$ is the tolerance assigned to the $j$ th locator at station $k$, and $w_{j, k}^{D}$ is a weighting coefficient related to its fabrication and assembly cost.

\subsubsection{Fixture maintenance cost}

The second group of manufacturing costs defines the cost due to the fixture maintenance. For a new fixture, the allowable varying ranges of locators position are determined by their design tolerances. Thus, the $j$ th locator of a manufactured fixture should match an initial precision defined by the design tolerance $\Delta l_{j, k}^{D}$. However, due to the gradual degradation, the dimension of a locator in station $k$ will decrease until it reaches a limit specified by the admissible maximum wear $\Delta l_{k}^{w}$. Then, a maintenance operation should be carried out. Such operations are commonly referred to as hard-time maintenance actions in industry. The time between fixture maintenance actions is defined by $t_{k}^{a}$, which can be estimated from maintenance data or fixture specifications.

Intuitively, a low frequency of maintenance actions imposes a higher precision of the initial locator dimensions (design tolerances) to keep parts within specifications for a longer period of time and thus, higher precision costs and lower fixture maintenance costs.

According to [14], the long-run average maintenance cost of a fixture at station $k$ can be expressed as:

$$
C_{k}^{M}=\frac{\left(\sum_{j=1}^{6} C_{j, k}^{L}\right)+C_{k}^{f}}{t_{k}^{a}},
$$

where

$$
\begin{aligned}
t_{k}^{a} & =\min _{j=1, \ldots, 6}\left(t_{k}^{a_{j}}\right), \\
t_{k}^{a_{j}} & =f_{k}^{j}\left(\Delta l_{k}^{w}\right), \quad k=1, \ldots, N, \quad j=1, \ldots, 6 ;
\end{aligned}
$$

$C_{k}^{f}$ is the fixed cost related to the maintenance operations at station $k$; and $t_{k}^{a_{j}}$ is the time required to wear the $j$ th locator at station $k$ up to a level of $\Delta l_{k}^{w}$, and it is modeled by the function $f_{k}^{j}(\cdot)$. According to the nature of machining processes, and without a loss of generality, the following assumptions are made in regarding to the progressive degradation in locators. In fixture devices based on punctual locators, material wear rates are constant along the time as it can be observed in many maintenance handbooks (e.g. [27]). However, fixture components are usually coated with special materials to minimize the wear rate. Thus, the wear rates of fixture devices usually presents a very low wear rate at the beginning of the production but increases when the coating is abrased or deteriorated. Therefore, for the sake of simplicity, the fixture degradation can be modeled as a quadratic curve with respect to the production time. Thus, the admissible maximum wear of fixture locators at station $k$ can be modeled as $\Delta l_{k}^{w}=G_{k}\left(t_{k}^{a}\right)^{2}$, where $G_{k}$ is a constant that indicates the wear rate of locators at station $k$ and $t_{k}^{a}$ is the operation time when a fixture maintenance action is conducted. According to this definition, the locator wear defined as $\Delta l_{k}^{w}$ is a random variable with the pdf derived in Appendix A. 


\subsubsection{Cutting-tool replacement cost}

A cutting-tool replacement is conducted when the admissible maximum wear value of the cuttingtool is reached. The time to replace the $\tau$ th cutting-tool at station $k$, called $t_{\tau, k}^{b}$, basically depends on two factors: i) the deviation generated on the machined feature due to the cutting-tool wear, and ii) the maximum flank wear recommended by the tool manufacturing vendor to avoid breakage or unsafe operations. In general, two terms define the cutting-tool replacement cost. The first term refers to the residual cost of the cutting-tool which is the cost of under-using the cutting-tool due to an early replacement before the maximum cutting-tool wear is reached. The second term refers to the cost of the cutting-tool replacement itself that involves the replacement of cutting-inserts or cutting-tools by operators.

Intuitively, a high replacement frequency decreases the dimensional variability of the machined features but increases the replacement cost. Therefore, cutting-tool replacement policies seek a tradeoff between the dimensional variability of the feature machined due to tool-wear and the replacement cost of the cutting-tool. In this paper, the cost of cutting-tool replacement is defined as follows:

$$
C_{\tau, k}^{w}=\left(w_{k}^{r e s_{\tau}} \cdot R_{\tau, k}+w_{k}^{r e p_{\tau}}\right) / t_{\tau, k}^{b}
$$

where

$$
\begin{aligned}
R_{\tau, k} & =\min _{\varphi=1, \ldots, \mu_{\tau, k}}\left(\frac{\left(V_{\tau, k}^{\max _{\varphi}}-V_{\tau, k}^{\varphi}\right)}{V_{\tau, k}^{\max _{\varphi}}}\right) \\
t_{\tau, k}^{b} & =\min _{\varphi=1, \ldots, \mu_{\tau, k}}\left(t_{\tau, k}^{b_{\varphi}}\right), \\
t_{\tau, k}^{b_{\varphi}} & =g_{k}\left(V_{\tau, k}^{\varphi}\right), \quad k=1, \ldots, N
\end{aligned}
$$

where $w_{k}^{r e s_{\tau}}$ is the residual cost of under-using a cutting-tool edge in the $\tau$ th cutting-tool at station $k ; w_{k}^{r e p_{\tau}}$ is the cost related to replace or turn the $\tau$ th cutting-tool in order to use a new cutting-tool edge at station $k ; R_{\tau, k}$ is the residual life of the cutting-tool in terms of percentage; $t_{\tau, k}^{b_{\varphi}}$ is the time required to wear the $\varphi$ th edge of the $\tau$ th cutting-tool at station $k$ up to a flank wear value of $V_{\tau, k}^{\varphi}$, and it is modeled by the function $g_{k}(\cdot)$; and $V_{\tau, k}^{\max \varphi}$ is the admissible maximum tool wear in the $\varphi$ th edge of the $\tau$ th cutting-tool to avoid a breakage or an unsafely machining operation at station $k$. It is noted that $\tau, \tau=1, \ldots, \kappa_{k}$, is the index of cutting-tools used at station $k$, and $\varphi, \varphi=1, \ldots, \mu_{\tau, k}$, is the index of cutting edges, which remove material simultaneously (e.g. end mills use two cutting edges simultaneously). In the literature, cutting-tool wear has been modeled as a third-order polynomial function of machining time in common machining operations, whereas in machining operations with high cutting speeds the cutting-tool wear tends to follow a second-order function [28]. Without loss of generality, it is assumed that the cutting-tool flank wear curve follows a second-order polynomial function with machining time. Thus, the admissible maximum wear of the $\tau$ th cutting-tool at the station $k$ can be modeled as $V_{\tau, k}^{\varphi}=E_{\tau, k}^{\varphi} \cdot t_{k}^{b}+F_{\tau, k}^{\varphi} \cdot\left(t_{k}^{b}\right)^{2}$, where $t_{k}^{b}$ is the machining time when a cutting-tool replacement is conducted. For this equation, $E_{\tau, k}^{\varphi}$ and $F_{\tau, k}^{\varphi}$ are coefficients that model the wearing rate of the $\varphi$ th cutting-tool edge of the $\tau$ th cutting-tool at station $k$. According to this definition, the cutting-tool wear, defined as $V_{\tau, k}^{\varphi}$, is a random variable with the pdf derived in Appendix A. 


\subsubsection{Quality loss cost}

According to [29], even if a product is well within specifications, the product has a quality loss if its quality characteristic value is not at the ideal performance target. In manufacturing, the quality loss associated may include the cost of scrap or rework. As this paper deals with variation propagation and the SoV model predicts the variability of the KPCs, the ideal targets value of the KPC variations are zeros, which refer to no-variation is presented. The Taguchi quality loss function for this case is the "smaller-the-better" function [29]. The equation used to describe this quality loss function of one part is:

$$
L=\frac{I_{0} \cdot y_{i}^{2}}{\left(T_{y_{i}} / 2\right)^{2}},
$$

where $I_{0}$ is the loss in monetary units per unit of time when the $0.27 \%$ of parts manufactured are out of specifications; $y_{i}$ is the deviation of the $i$ th KPC from its target; and $T_{y_{i}}$ is the dimensional tolerance of the $i$ th KPC.

\subsection{Definition of constraints}

\subsubsection{Constraints of non-maintainable KCCs}

Many KCCs that contribute to final KPC variations can not be cost-effectively maintained or controlled since their variation ranges cannot be reduced through correction actions in an feasible way. However, although the variation range of these KCCs, named non-maintainable KCCs, cannot be reduced, they can be estimated when shop-floor data is available. In the process-oriented tolerancing problem, the variability of each non-maintainable $K C C$ is restricted to a constant range. For instance, one common non-maintainable $K C C$ is generally related to the temperature variations of the machinetool spindle. In practice, it is infeasible to maintain or control the temperature of the machine-tool spindle during machining. However one can estimate, using historical data, the temperature variation range of the machine-tool in normal conditions. Thus, the deviation of the spindle temperature with respect to the nominal temperature can be restricted by the six-sigma range as:

$$
-3 \cdot \sigma_{T_{k}^{s}} \leq \Delta T_{k}^{s} \leq+3 \cdot \sigma_{T_{k}^{s}}, \quad k=1, \ldots, N,
$$

where $\sigma_{T_{k}^{s}}$ is the standard deviation of the spindle temperature at station $k$ under normal manufacturing conditions, which is estimated from empirical shop-floor data or a priori knowledge. Other nonmaintainable KCCs such as cutting-tool deflections due to cutting-force variations or geometrical axis deviations due to straightness errors or misalignments can be defined by similar constraints.

\subsubsection{Constraints of maintainable KCCs}

Unlike non-maintainable KCCs, the maintainable KCCs can be kept within a range of variation by adequate corrective actions. Two common types of maintainable KCCs are related to the initial precision of fixture locators and the wear of cutting-tools. Constraints of these maintainable KCCs are: i) the initial precision of locators cannot be lower than a minimum tolerance, denoted by $\Delta l_{\text {min }}^{D}$, which is defined by manufacturing and assembly limitations of fixture components; ii) the initial precision of locators, in case that a non-accurate locator were needed, cannot be higher than a maximum tolerance range, denoted by $\Delta l_{\max }^{D}$; and iii) the maximum cutting-tool wear cannot exceed its maximum allowable value, denoted by $V_{\tau, k}^{\max }$, which is set to avoid cutting-tool breakage or unsafely operations. These constraints can be written as: 


$$
\begin{aligned}
& 0 \leq V_{\tau, k}^{\varphi} \leq V_{\tau, k}^{\max _{\varphi}} \\
& \Delta l_{\min }^{D} \leq \Delta l_{j, k}^{D} \leq \Delta l_{\max }^{D}
\end{aligned}
$$

for $k=1, \ldots, N, j=1, \ldots, 6, \tau=1, \ldots, \kappa_{k}$ and $\varphi=1, \ldots, \mu_{\tau, k}$.

\subsubsection{Part quality constraints}

Process tolerances will be set in order to ensure part design specifications. For this purpose, any part design specification should be mathematically expressed by constraint equations. A widely accepted approach is transforming the GD\&T specifications shown in part design drawings into VD\&T specifications $[4,30,31]$, defining the maximum values of the DMVs for the toleranced feature. For position tolerance zones on planar surfaces, the following methodology to translate a GD\&T specification into constraints of DMVs can be applied:

- Step 1: Identify the controlledfeature $\left(S_{q}\right)$, the measurement datum feature $\left(S_{D}\right)$ and the GD\&T reference datum feature $\left(S_{m}\right)$. A controlled feature is defined as the toleranced feature whose position tolerance needs to be translated into constraints. A measurement datum feature refers to the feature used as a datum in the inspection station where the inspection of part quality is conducted. A GD\&T reference datum feature is the datum feature applied in the toleranced feature definition.

- Step 2: Identify the boundary points $\left\{P_{1}^{S_{q}}, \ldots, P_{P}^{S_{q}}\right\}$ that define the controlled feature. For a plane, its $P$ extreme points are considered as the boundary points since they are most likely to be out of specifications due to orientation deviations.

- Step 3: Determine the quality constraints according to the type of measurement conducted to verify the specifications. These measurements can be direct, when the GD\&T reference datum feature and the measurement datum feature are the same, or indirect, when they are different. For each type of measurements, the constraints are defined as follows:

(a) Specification verified by direct measurements. According to the SoV model in Eq. (3), if we assume that measurement and linearization errors in comparison with part dimension deviations are negligible, the variability of the LCS of the controlled feature $S_{q}$ is defined by the covariance matrix:

$$
\Sigma_{\mathbf{Y}_{S_{q}}}=\Gamma_{S_{q}} \cdot \Sigma_{\mathbf{U}} \cdot \Gamma_{S_{q}}^{T},
$$

where $\Sigma_{\mathbf{U}}$ is the covariance matrix of the $s$ independent process variables defined by the vector $\mathbf{U}$ (e.g. the locator deviation due to its initial precision and its degradation, cuttingtool wear, etc.), and it has the form $\operatorname{diag}\left\{\operatorname{Var}\left(u_{1}\right), \ldots, \operatorname{Var}\left(u_{s}\right)\right\}$ where $\operatorname{Var}(\bullet)$ is the variance of $\bullet$; and $\Gamma_{S_{q}}$ is a block matrix of $\Gamma$ that corresponds to the deviation of feature $S_{q}$, obtained from Eq. (3). From Eq. (17), the variability of the boundary point $P_{p}$ in the controlled feature $S_{q}$ can be defined as:

$$
\Sigma_{\mathbf{Y}_{P_{p}}^{S_{q}}}=\left(\mathbf{C}_{P_{p}} \cdot \boldsymbol{\Gamma}_{S_{q}}\right) \cdot \Sigma_{\mathbf{U}} \cdot\left(\mathbf{C}_{P_{p}} \cdot \boldsymbol{\Gamma}_{S_{q}}\right)^{T}
$$


where matrix $\mathbf{C}_{P_{p}}$ is defined in Appendix B. According to the assumptions in Section 3.1 and the Lindeberg's central limit theorem $[32,33]$, the variability of the KPCs can be approximated to a normal distribution if a large number of process variables is considered. Thus, the variability constraints of each boundary point at the direction where the tolerance applies can be defined as follows:

$$
\Sigma_{\mathbf{Y}_{P_{p}}^{S_{q}}}(\rho, \rho)=\left(\mathbf{C}_{P_{p}} \cdot \boldsymbol{\Gamma}_{S_{q}}\right) \cdot \Sigma_{\mathbf{U}} \cdot\left(\mathbf{C}_{P_{p}} \cdot \boldsymbol{\Gamma}_{S_{q}}\right)^{T}(\rho, \rho) \leq\left(\frac{T}{6}\right)^{2}, \quad \forall P_{p} \in S_{q},
$$

where $\Sigma_{(\cdot)}(\rho, \rho)$ refers to the $(\rho, \rho)$ element of the covariance matrix $\Sigma_{(\cdot)} ; \rho=1,2,3$ according to the direction where the position tolerance applies (e.g. $\rho=1$ for $x$ direction, etc.); and $T$ is the tolerance for this position tolerance specification.

(b) Specification verified by indirect measurements. For this case, the variability of the boundary point $P_{p}$ in the controlled feature $S_{q}$ is defined by the covariance matrix:

$$
\Sigma_{\mathbf{Y}_{P_{p}}^{S_{q}}}=\left(\mathbf{C}_{P_{p}} \cdot \mathbf{K} \cdot\left[\begin{array}{ll}
\boldsymbol{\Gamma}_{S_{q}} & \boldsymbol{\Gamma}_{S_{m}}
\end{array}\right]^{T}\right) \cdot \Sigma_{\mathbf{U}} \cdot\left(\mathbf{C}_{P_{p}} \cdot \mathbf{K} \cdot\left[\begin{array}{ll}
\boldsymbol{\Gamma}_{S_{q}} & \boldsymbol{\Gamma}_{S_{m}}
\end{array}\right]^{T}\right)^{T},
$$

where matrix $\mathbf{K}$ is defined in Appendix $\mathbf{C}$. Thus, the variability constraints of each boundary point at the direction where the tolerance applies can be defined as follows:

$$
\begin{aligned}
\Sigma_{\mathbf{Y}_{P_{p}}^{S_{q}}}(\rho, \rho)=\left(\mathbf{C}_{P_{p}} \cdot \mathbf{K} \cdot\left[\begin{array}{ll}
\boldsymbol{\Gamma}_{S_{q}} & \boldsymbol{\Gamma}_{S_{m}}
\end{array}\right]^{T}\right) \cdot \Sigma_{\mathbf{U}} \cdot\left(\mathbf{C}_{P_{p}} \cdot \mathbf{K} \cdot\left[\begin{array}{ll}
\boldsymbol{\Gamma}_{S_{q}} & \boldsymbol{\Gamma}_{S_{m}}
\end{array}\right]^{T}\right)^{T}(\rho, \rho) \leq\left(\frac{T}{6}\right)^{2} \\
\forall P_{p} \in S_{q}
\end{aligned}
$$

Eq. (19) and Eq. (21) are quality constraints for a boundary point $P_{p}$ in the controlled feature $S_{q}$ in case that the inspection is conducted by a direct or indirect measurement respectively. If the controlled feature is defined with $p=1, \ldots, P$ boundary points, then $P$ quality constraints will be defined. As a general form, the quality constraints are defined by the inequality:

$$
D_{p}^{q} \leq\left(\frac{T_{q}}{6}\right)^{2}, \quad \forall P_{p} \in S_{q}
$$

where $T_{q}$ is the tolerance specification of the controlled feature $S_{q}$, and the term $D_{p}^{q}$ is defined as shown above according to the type of measurement conducted to verify the part specification.

\subsection{Optimization model}

As presented above, the process-oriented tolerancing becomes the generic optimization problem defined in Eq. (4). Its resolution assigns the tolerances of the maintainable KCCs in order to make the manufacturing cost minimum and keep the parts within specifications. Based on the set of various costs defined in previous subsections, this optimization problem can be re-formulated as:

$$
\begin{aligned}
& \min _{\Delta l_{j, k}^{D}, \Delta l_{k}^{w}, V_{\tau, k}^{\varphi}}\left[\sum_{k=1}^{N} w_{k}^{M} \cdot \frac{\left(\sum_{j=1}^{6} \frac{w_{j, k}^{D}}{\Delta l_{j, k}^{D}}\right)+C_{k}^{f}}{t_{k}^{a}}+\right. \\
& \left.+\sum_{k=1}^{N} \sum_{\tau=1}^{\kappa_{k}} w_{k}^{\tau} \cdot\left(w_{k}^{r e s_{\tau}} \cdot \min _{\varphi=1, \ldots, \mu_{\tau, k}}\left(\frac{\left(V_{\tau, k}^{\max _{\varphi}}-V_{\tau, k}^{\varphi}\right)}{V_{\tau, k}^{\max \varphi}}\right) / t_{\tau, k}^{b}+\frac{w_{k}^{r e p_{\tau}}}{t_{\tau, k}^{b}}\right)+\sum_{i=1}^{M} \frac{I_{0} \cdot y_{i}^{2}}{\left(T_{y_{i}} / 2\right)^{2}}\right]
\end{aligned}
$$


subjected to:

$$
\begin{aligned}
& -3 \cdot \sigma_{T_{k}^{s}} \leq \Delta T_{k}^{s} \leq+3 \cdot \sigma_{T_{k}^{s}}, \\
& 0 \leq V_{\tau, k}^{\varphi} \leq V_{\tau, k}^{\max }, \\
& \Delta l_{\min }^{D} \leq \Delta l_{j, k}^{D} \leq \Delta l_{\max }^{D}, \\
& D_{p}^{q} \leq\left(\frac{T_{q}}{6}\right)^{2},
\end{aligned}
$$

where $j=1, \ldots, 6 ; k=1, \ldots, N ; i=1, \ldots, M ; q=1, \ldots, Q ; p=1, \ldots, P ; \varphi$ refers to the cutting edge of the $\tau$ th cutting-tool at the $k$ th station with $\varphi=1, \ldots, \mu_{\tau, k}$ and $\tau=1, \ldots, \kappa_{k} ; D_{p}^{q}$ refers to the constraints due to position tolerance specifications; $T_{q}$ the tolerance value applied to the controlled feature; and $w_{k}^{M}$ and $w_{k}^{\tau}$ are weighting coefficients for the costs related to fixture maintenance actions and cutting-tool replacements respectively. The optimization problem is summarized in Fig. 3.

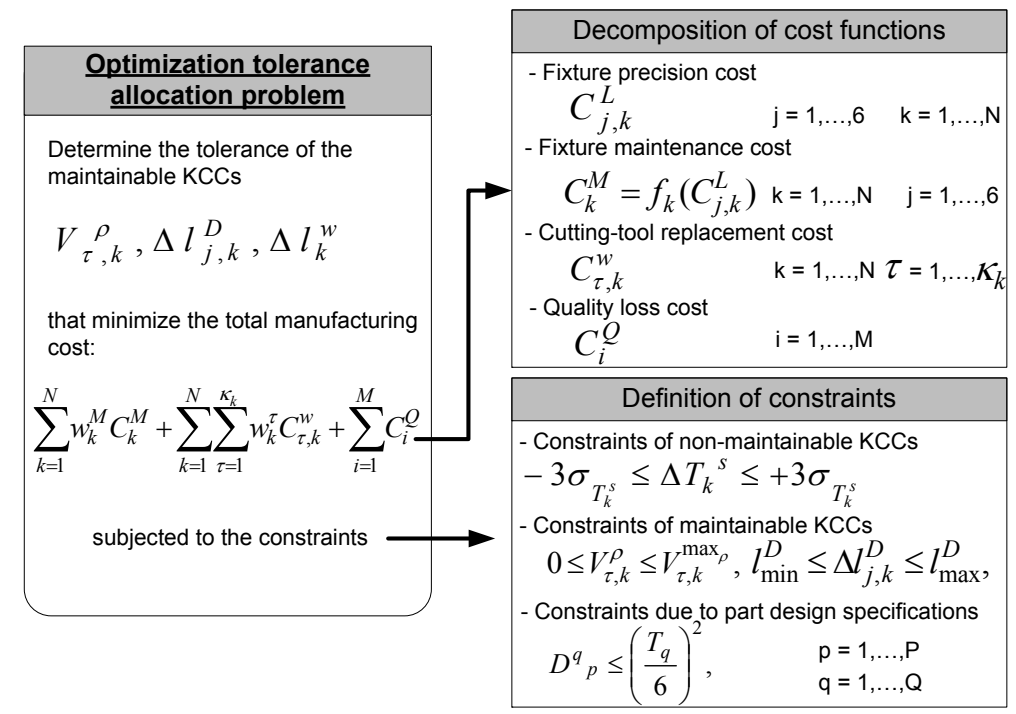

Figure 3: Process-oriented tolerancing.

\section{Case Study}

\subsection{Problem description}

To demonstrate the proposed process-oriented tolerancing methodology, we conducted a case study that manufactures parts, as shown in Fig. 4, with a 3-station machining process, as illustrated in Fig. 5. The datum features and the surfaces to be machined are summarized in Table 1. The position of fixture locators at each machining station are defined in Table 2. The KPCs of the part are the normal distance between $S_{3}$ and $S_{0}$, denoted as $K P C_{1}$, and the normal distance between $S_{8}$ and $S_{6}$, denoted as $\mathrm{KPC}_{2}$. The position tolerance requirements for both KPCs are $\pm 0.05 \mathrm{~mm}$. The extended SoV model presented in [11] is applied to model the variation propagation along this MMP. In this extended model, the KCCs modeled are the deviation of the fixture locators, the wear of the cuttingtools and the spindle thermal expansion of the machine-tools. For this case study, the process-oriented 
Table 1: Process planning. Features machined at each station and datum features based on the 3-2-1 fixture schemes Datum Features

\begin{tabular}{cccc} 
Station & features & machined & Cutting-tool \\
\hline 1 & $S_{0}-S_{4}-S_{5}$ & $S_{2}$ & ADHX 110305, PVD TiAlN $\phi=24.856 \mathrm{~mm}, L=111.322 \mathrm{~mm}$ \\
2 & $S_{2}-S_{6}-S_{5}$ & $S_{1}$ & ADHX 110305, PVD TiAlN $\phi=24.856 \mathrm{~mm}, L=111.322 \mathrm{~mm}$ \\
3 & $S_{1}-S_{4}-S_{5}$ & $S_{3}, S_{8}$ & ADHX 110305, PVD TiAlN $\phi=24.856 \mathrm{~mm}, L=111.322 \mathrm{~mm}$ \\
4 & $S_{0}-S_{4}-S_{5}$ & & Inspection station \\
\hline
\end{tabular}

$\phi:$ Tool diameter; $L:$ Tool length

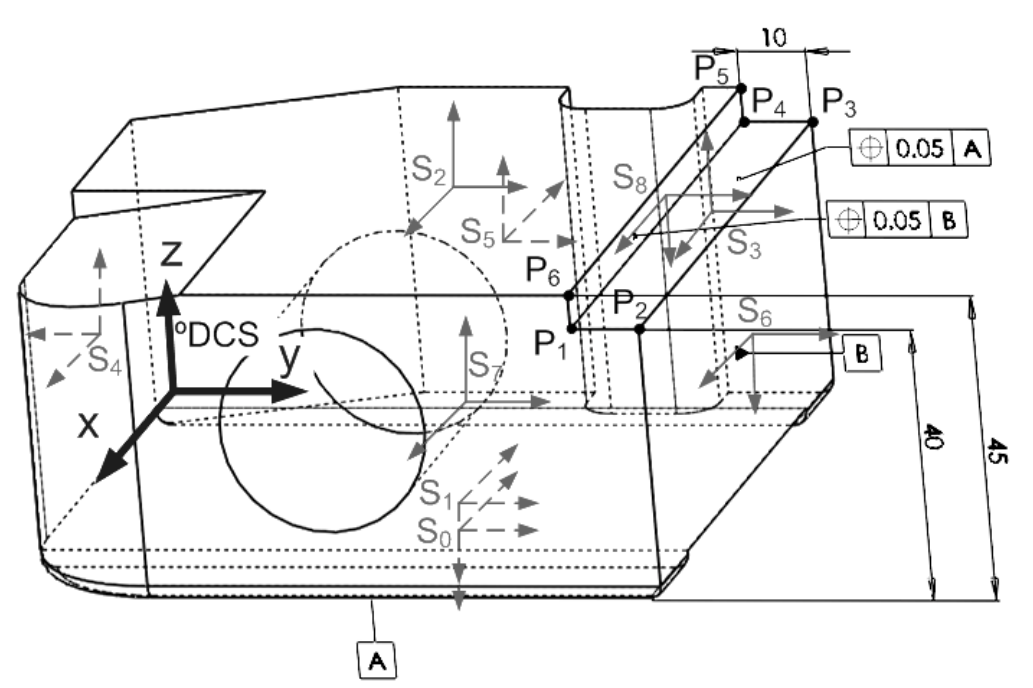

Figure 4: Final machined part for the case study (unit: $\mathrm{mm}$ ).

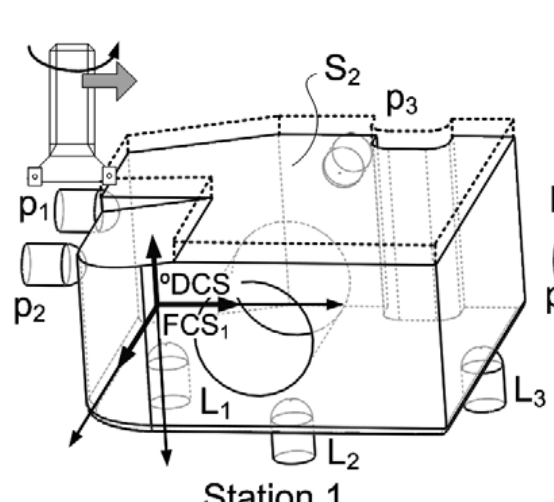

Station 1

(datums $\mathrm{S}_{0}-\mathrm{S}_{4}-\mathrm{S}_{5}$ )

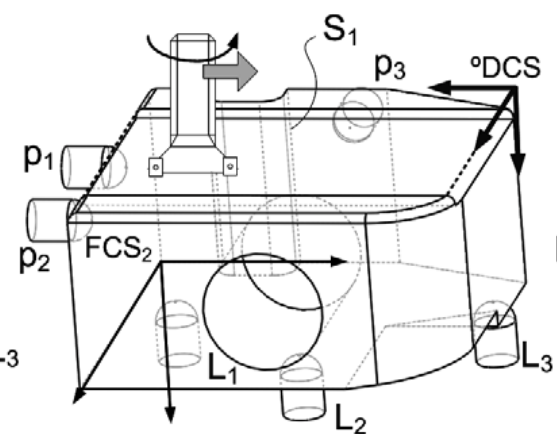

Station 2

(datums $\mathrm{S}_{2}-\mathrm{S}_{6}-\mathrm{S}_{5}$ )

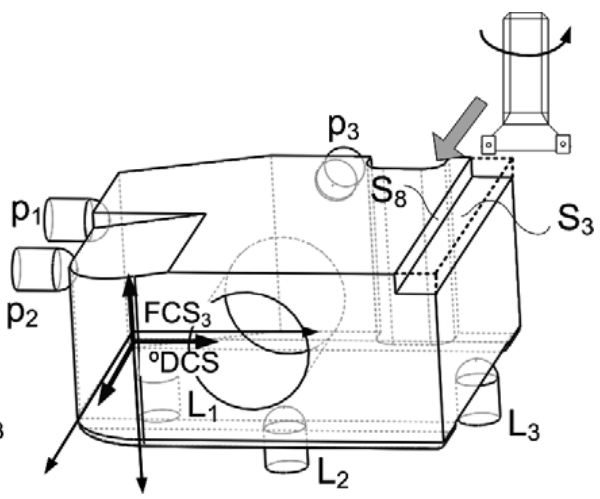

Station 3

(datums $\mathrm{S}_{1}-\mathrm{S}_{4}-\mathrm{S}_{5}$ )

Figure 5: Case study. Part to be machined through 3 machining stations. 
Table 2: Fixture layout.

\begin{tabular}{cccc} 
Station & $\left(\mathbf{w}_{F_{k}}^{\circ}\right)^{T}(-\mathrm{rad}-)$ & $\left(\mathbf{t}_{F_{k}}^{\circ}\right)^{T}(-\mathrm{mm}-)$ & Locators w.r.t. FC $S_{k}(-\mathrm{mm}-)$ \\
\hline 1 & {$[-\pi / 2, \pi, 0]$} & {$[0,0,0]$} & $L_{1 x}=10, L_{1 y}=30, L_{2 x}=50, L_{2 y}=70, L_{3 x}=90, L_{3 y}=30$ \\
& & & $p_{1 y}=30, p_{1 z}=-35, p_{2 y}=70, p_{2 z}=-35, p_{3 x}=50, p_{3 z}=-20$ \\
2 & {$[\pi / 2,0,0]$} & {$[0,95,45]$} & $L_{1 x}=10, L_{1 y}=30, L_{2 x}=50, L_{2 y}=70, L_{3 x}=90, L_{3 y}=30$ \\
& & & $p_{1 y}=30, p_{1 z}=-35, p_{2 y}=70, p_{2 z}=-35, p_{3 x}=50, p_{3 z}=-20$ \\
3 & {$[-\pi / 2, \pi, 0]$} & {$[0,0,2.5]$} & $L_{1 x}=10, L_{1 y}=30, L_{2 x}=50, L_{2 y}=70, L_{3 x}=90, L_{3 y}=30$ \\
& & & $p_{1 y}=30, p_{1 z}=-35, p_{2 y}=70, p_{2 z}=-35, p_{3 x}=50, p_{3 z}=-20$ \\
\hline
\end{tabular}

Table 3: Nominal location and orientation of each feature.

\begin{tabular}{ccc} 
Feature & $\left(\mathbf{w}_{S_{i}}^{D}\right)^{T}(-\mathrm{rad}-)$ & $\left(\mathbf{t}_{S_{i}}^{\circ}\right)^{T}(-\mathrm{mm}-)$ \\
\hline$S_{0}$ & {$[0, \pi, 0]$} & {$[47.5,47.5,0]$} \\
$S_{1}$ & {$[0, \pi, 0]$} & {$[47.5,47.5,2.5]$} \\
$S_{2}$ & {$[0,0,0]$} & {$[47.5,42.5,45]$} \\
$S_{3}$ & {$[0,0,0]$} & {$[47.5,90,40]$} \\
$S_{4}$ & {$[\pi / 2, \pi / 2,-\pi / 2]$} & {$[47.5,0,22.5]$} \\
$S_{5}$ & {$[0, \pi / 2,0]$} & {$[0,47.5,22.5]$} \\
$S_{6}$ & {$[\pi / 2,-\pi / 2,-\pi / 2]$} & {$[47.5,95,20]$} \\
$S_{7}$ & {$[-\pi / 2,-\pi / 2,0]$} & {$[95,47.5,22.5]$} \\
$S_{8}$ & {$[\pi / 2,-\pi / 2,-\pi / 2]$} & {$[47.5,85,42.5]$} \\
\hline
\end{tabular}

tolerancing is formulated as:

$$
\begin{aligned}
& \min _{\Delta l_{j, k}^{D}, \Delta l_{k}^{w}, V_{1, k}^{\varphi}}\left[\sum_{k=1}^{3} w_{k}^{M} \cdot \frac{\left(\sum_{j=1}^{6} \frac{w_{j, k}^{D}}{\Delta l_{j, k}^{D}}\right)+C_{k}^{f}}{t_{k}^{a}}\right. \\
& \left.+\sum_{k=1}^{3} w_{k}^{1} \cdot\left(w_{k}^{r e s_{1}} \cdot \min _{\varphi=1,2}\left(\frac{\left(V_{1, k}^{\max \varphi}-V_{1, k}^{\varphi}\right)}{V_{1, k}^{\max }}\right) / t_{1, k}^{b}+\frac{w_{k}^{r e p_{1}}}{t_{1, k}^{b}}\right)+\sum_{i=1}^{2} \frac{I_{0} \cdot y_{i}^{2}}{\left(T_{y_{i}} / 2\right)^{2}}\right],
\end{aligned}
$$

for $j=1, \ldots, 6 ; k=1,2,3 ; i=1,2$ and $\varphi=1,2$. We use one cutting-tool at each station, and each cutting-tool has two cutting edges $(\varphi=1,2)$. Furthermore, the two edges of the cutting-tool are only used at station 3 (end milling operation), while in the first two stations, only one cutting edge is used (face milling operations). This cost function should be minimized subjected to the following 15 constraints:

- 3 constraints on the admissible thermal variation of the machine-tool spindle at each station. As these process variables are non-maintainable KCCs, it is assumed that the specific thermal variation range at each station in normal conditions is known from the shop-floor knowledge.

- 4 constraints on the maximum cutting-tool wear, one for each cutting-tool edge used. Note that in the third station, primary and secondary cutting-tool edges are used to machine the features $S_{8}$ and $S_{3}$. Thus, two constraints should be defined at that station.

- 4 constraints on the position tolerance of the $K P C_{1}$, one constraint for each point that defines the boundaries of the $S_{3}$ plane (points $P_{1}, P_{2}, P_{3}, P_{4}$ ). 
Table 4: Numerical values for manufacturing tolerance allocation in the case study.

\begin{tabular}{|c|c|c|c|c|c|c|c|}
\hline \multicolumn{4}{|c|}{ STATION $1 \& 2(k=1,2)$} & \multicolumn{4}{|c|}{ STATION $3(k=3)$} \\
\hline Parameter & Value & Parameter & Value & Parameter & Value & Parameter & Value \\
\hline$w_{i, k}^{D}$ & $3 \mathrm{u}$ & $C_{k}^{f}$ & $500 \mathrm{u}$ & $w_{i k}^{D}$ & $3 \mathrm{u}$ & $C_{k}^{f}$ & $500 \mathrm{u}$ \\
\hline$w_{k}^{r e s} s_{1}$ & $20 \mathrm{u}$ & $w_{k}^{r e p_{1}}$ & $10 \mathrm{u}$ & $w_{k}^{\text {reses }}$ & $20 \mathrm{u}$ & $w_{k}^{r e p_{1}}$ & $10 \mathrm{u}$ \\
\hline$w_{k}^{M}$ & 1 & $w_{k}^{1}$ & 1 & $w_{k}^{M}$ & 1 & $w_{k}^{1}$ & 1 \\
\hline$V_{1, k}^{m a n}$ & $0.4 \mathrm{~mm}$ & $G_{k}^{k}$ & $1.9 \cdot 10^{-7} \frac{\mathrm{mm}}{\mathrm{hr} r^{2}}$ & $V_{1, k}^{\max _{1}}$ & $0.4 \mathrm{~mm}$ & $V_{1, k}^{k} \max _{2}$ & $0.4 \mathrm{~mm}$ \\
\hline$E_{1, k}^{1}$ & $0.0113 \frac{\mathrm{mm}}{\mathrm{hr}}$ & $F_{1, k}^{1}$ & $0.0019 \frac{\mathrm{mm}}{h r^{2}}$ & $G_{k}$ & $1.9 \cdot 10^{-7} \frac{\mathrm{mm}}{\mathrm{hr}^{2}}$ & $E_{1, k}^{1, n} \& E_{1, k}^{2}$ & $0.0113 \frac{\mathrm{mm}}{\mathrm{hr}}$ \\
\hline$\Delta l_{\min }^{D}$ & $0.010 \mathrm{~mm}$ & $\Delta l_{\max }^{D}$ & $0.4 \mathrm{~mm}$ & $F_{1, k}^{1} \& F_{1, k}^{2}$ & $0.0019 \frac{\mathrm{mm}}{h r^{2}}$ & $\pm 3 \sigma_{T_{k}^{s}}$ & $\pm 5^{\circ} \mathrm{C}$ \\
\hline $\pm 3 \sigma_{T_{k}^{s}}$ & $\pm 5^{\circ} \mathrm{C}$ & & & $\Delta l_{\min }^{D}$ & $0.010 \mathrm{~mm}$ & $\Delta l_{\max }^{D}$ & $0.4 \mathrm{~mm}$ \\
\hline
\end{tabular}

Table 5: Parameters applied in the GA and the MADS algorithm

Genetic Algorithm (GA)

\begin{tabular}{llll}
\hline Parameter & Value & Parameter & Value \\
\hline Variables to optimize & 24 & Initial Population & 60 \\
Initial Population Distributions & & Reproduction Options & \\
$\quad \Delta l_{k}^{w},(\forall k)$ & Unif $(0.002,0.040)$ & Elite count & 10 \\
$\quad \Delta l_{j, k}^{D},(\forall j, \forall k)$ & Unif $(0.010,0.040)$ & Crossover fraction & 0.8 \\
$\quad V_{1, k}^{\varphi},(\forall \varphi, \forall k)$ & Unif $(0,0.4)$ & Crossover function & Heuristic (ratio=1.2) \\
Scaling Function & Rank & Mutation function & Uniform $(\mathrm{P}=0.05)$ \\
Selection Options & Roulette & Stop criterium & Generations: 15 \\
\hline & & & \\
Mesh Adaptive Direct Search Algorithm (MADS) & & \\
\hline Parameter & Value & Parameter & Value \\
\hline Variables to optimise & 24 & Contraction & 0.5 \\
Initial Mesh Size & 1 & Poll Method & Positive Basis 2N \\
Max. Mesh Size & Inf & Polling order & Consecutive \\
Max. Func. Eval. & Inf & Stop criterium & Tol. mesh: $8 \cdot 10^{-3}$ \\
Expansion & 2 & & \\
\hline
\end{tabular}

- 4 constraints on the position tolerance of the $K P C_{2}$, one constraint for each point that defines the boundaries of the $S_{8}$ plane (points $P_{1}, P_{4}, P_{5}, P_{6}$ ).

For the sake of simplicity, it is assumed that all locators are degraded identically along the manufacturing process.

\subsection{Numerical analysis}

A numerical case study was conducted with the values presented in Table 4. The optimization problem with 24 variables (18 variables for locator design tolerances, i.e. $\left\{\Delta l_{j, k}^{D}\right\} ; 3$ variables for admissible maximum wear of fixture locators, i.e. $\left\{\Delta l_{k}^{w}\right\}$; and 3 variables for the admissible maximum wear of each cutting-tool edge, i.e. $\left.\left\{V_{1, k}^{\varphi}\right\}\right)$ and 15 constraints is difficult to be solved using traditional optimization techniques, requiring other non-traditional techniques [34]. For this case study, two algorithms were applied sequentially. Firstly, a genetic algorithm (GA) was run in Matlab ${ }^{\circledR}$ from an initial population of 60 individuals for 15 generations in order to find a region close to the optimal solution. The initial population was created randomly by uniform distributions. Secondly, the solution provided by the GA algorithm was used as the initial point in a mesh adaptive direct search (MADS) algorithm for tuning the optimal result. The MADS algorithm was run until the tolerance mesh reached a value of $8 \cdot 10^{-3}$. The optimization procedure was repeated five times to ensure the 
convergence of the solution. Table 5 shows the parameters applied for both optimization algorithms according to the recommendations of the optimization toolbox user's guide from Matlab ${ }^{\complement}$ [35].

\subsection{Comparison with a conventional process-oriented tolerancing methodology}

The case study was analyzed comparing two process-oriented approaches: i) the proposed methodology considering additional process variables such as cutting-tool wear and thermal spindle expansion; and ii) a process-oriented tolerancing methodology based on the conventional SoV model (without machining process variables). The latter will be named conventional methodology hereafter. In the conventional methodology, the effects of other KCCs such as the cutting-tool wear and the thermal spindle expansion on part quality are not considered. The results of both methodologies are shown in Figs. 7-9. Note that tolerances of locators $p_{1}, p_{2}$ and $p_{3}$ at stations 2 and 3 and locator $p_{3}$ at station 3 are not shown since they do not affect any of the KPCs analyzed in this study and thus, they are set to the maximum tolerance range $\left(\Delta l_{\max }^{D}\right)$. In terms of costs, the proposed methodology estimates a manufacturing cost of $52.3 \mathrm{u} / \mathrm{hr}$, where the fixture maintenance cost, the cutting-tool replacement cost and the expected part quality loss cost are $24.4 u / h r, 17.3 u / h r$ and $10.6 u / h r$, respectively. On the other hand, the conventional methodology estimates a manufacturing cost of $21.0 \mathrm{u} / \mathrm{hr}$, where the fixture maintenance cost, cutting-tool replacement cost and the expected part quality loss cost are $11.2 u / h r, 2.7 u / h r$ and $7.1 u / h r$, respectively. Note that these results refer to estimations, but since it will be explained below, the estimations from the conventional methodology can be far away from the actual ones due to overlooking the effect of machining-induced variations on part quality.

As aforementioned, the objective of the proposed process-oriented tolerancing is to optimally assign the tolerance to the three components: (i) the fixture locators $\left(\Delta l_{j, k}^{D}\right.$ 's), (ii) the fixture maintenance threshold ( $\Delta l_{k}^{w}$ 's), and (iii) the cutting tool replacement threshold ( $V_{\tau, k}^{\max _{\varphi}}$ 's). The optimization is to achieve an overall minimum cost since these three components are directly related to four types of cost, i.e., the fixture precision cost, the fixture maintenance cost, the cutting-tool replacement cost and the part quality loss cost. Although both conventional and the proposed method for processoriented tolerancing consider these four cost components, subjected to the same quality constraints, the conventional method does not consider the impacts of the the cutting tool replacement threshold and the thermal expansion of the spindle on the part quality, since such impacts are not explicitly included in the SoV model. Thus, the quality loss cost and the quality constraints calculated by conventional methods may not be accurate enough to reflect the real part dimensional variation. Such model inaccuracy will result in two miscalculations in the conventional methodology. First, the part quality predicted by the conventional method would be better than the actual one, then the quality loss cost -Eq.(13)- would be lower and, therefore, looser tolerances than the required ones would be allocated. Second, the quality constraints -Eq.(22)- defined by the conventional method would not be restrictive enough since important machining-induced errors are not explicitly modeled. Thus, given a design tolerance from the design drawing, the tolerances allocated would be incorrectly wider than that should be since the tolerance will be assigned to less variables. The wider tolerance and mis-assigned contraints lead to lower cost of fixture maintenance and cutting-tool replacement. However, one can forsee that, according to this miscalculated process-oriented tolerance, the process will generate more non-conforming (out-of-specifications) parts than expected. Conversely, the proposed methodology are based on the SoV model that includes not only the fixture-induced variations but also machining-induced variation sources such as $\Delta T_{k}^{s}$ and $V_{\tau, k}^{\varphi}$ defined in $\mathbf{U}$ of the model -Eq.(3)-. Thus, the quality loss costs and the quality constraints are closer to the actual ones. Therefore, given a design tolerance from a design drawing, the proposed methodology tends to assign more tightened 
tolerances for all contributors ( $\Delta l_{j, k}^{D}$ 's, $\Delta l_{k}^{w}$ 's and $V_{\tau, k}^{\max \varphi}$ 's) since the manufacturing process should ensure that the parts are within specifications.

According to this theoretical elaboration, the proposed methodology will have higher fixture maintenance costs and cutting-tool replacement costs than the conventional methodology. However, the neglection of the machining-induced variation in the conventional methodology will result in more non-conforming parts, incurring a significantly higher part quality loss cost and thus, higher overall manufacturing cost.

In order to demonstrate the improvements of the proposed methodology over its conventional counterpart (i.e. the process-oriented tolerancing problem without considering machining-induced variations), 10,000 Monte Carlo simulations were run for two MMPs. The first and second MMP were defined according to the tolerances allocated using the conventional and the proposed approach, respectively. The model used to run the simulations is the extended SoV model derived experimentally in [11]. For each simulation, a random value for each $\mathrm{KCC}$ is calculated according to its pdf. For this case study, the initial locator precision is assumed normally distributed with mean 0 and standard deviation $\Delta l_{j, k}^{D} / 6$; the fixture wear and cutting-tool wear are random variables with the pdf derived in Appendix A. To simulate the effect of measurement noises and un-modeled sources of variation, a random variable normally distributed with mean 0 's and standard deviation $0.005 / 6 \mathrm{~mm}$ and $1.05 \cdot 10^{-3} / 6$ rad for dimensional and orientational noises, respectively, were added at each simulation. The simulation results concluded that the number of conforming parts (within specifications) was notably different for the two methodologies, indicating the importance of the incorporation of process variations, such as thermal and cutting-tool wear variations, in the process-oriented tolerancing. The proposed methodology kept the $99.21 \%$ of the parts conforming to the position specifications of $K P C_{1}$ and $K \mathrm{PC}_{2}$. However, the conventional methodology can only ensure a rate of $82.45 \%$ within specifications since the methodology assumes that machining-induced variations do not influence part quality, so looser tolerances are allocated while solving the optimization problem. Therefore, by including the cutting-tool wear and spindle thermal expansion effects on part quality, the process-oriented tolerance allocation improve the number of parts within specifications by $16.76 \%$. This result is also shown in the actual cost related to part quality loss. Although the expected quality loss is $10.6 \mathrm{u} / \mathrm{hr}$ and $7.1 \mathrm{u} / \mathrm{hr}$ for the proposed and conventional methodology respectively, both methodologies will present a higher quality loss cost due to the components added in the Monte Carlo simulation to simulate the effect of measurement noise and un-modeled components of the variation propagation itself. Besides these factors, the conventional methodology will present an additional component that will increase the actual quality loss cost since its variation propagation model does not considers the effect of cutting-tool wear or thermal variations, so one can expect a higher number of non-conforming parts (outside specifications). After Monte Carlo simulations, the actual quality loss for the proposed and the conventional methodology was $17.63 u / h r$ and $52.23 u / h r$, respectively. This means that the final manufacturing cost by allocating the tolerances of the KCCs with the proposed methodology is increased to $59.41 \mathrm{u} / \mathrm{hr}$, and with the conventional methodology, the manufacturing cost is increased to $66.16 u / h r$, a $11.36 \%$ higher than the proposed one. Table 6 shows the results after Monte Carlo simulations for both methodologies. Besides the comparison analysis, the Monte Carlo simulations were also analyzed to confirm that, for the case study, the Lindeberg's central limit theorem holds and the variability of the KPCs can be approximated to a normal distribution despite that some KCCs are not normally distributed. Fig. 6 shows the pdf of the deviation of feature $S_{3}$ in $Z$ direction according to Monte Carlo simulations and according to the approximation used in the paper assuming that Lin- 
Table 6: Actual cost values and part quality after Monte Carlo simulations according to the process-oriented tolerancing methodology applied

\begin{tabular}{lccccc} 
& $\begin{array}{c}\text { Fixture } \\
\text { maintenance cost }\end{array}$ & $\begin{array}{c}\text { Cutting-tool } \\
\text { replacement cost }\end{array}$ & $\begin{array}{c}\text { Part quality } \\
\text { loss cost }\end{array}$ & $\begin{array}{c}\text { Manufacturing } \\
\text { cost }\end{array}$ & $\begin{array}{c}\% \text { of parts } \\
\text { within specifications }\end{array}$ \\
\hline Proposed method. & $24.4 \mathrm{u} / \mathrm{hr}$ & $17.3 \mathrm{u} / \mathrm{hr}$ & $17.63 \mathrm{u} / \mathrm{hr}$ & $59.41 \mathrm{u} / \mathrm{hr}$ & $99.21 \%$ \\
Conventional method. & $11.2 \mathrm{u} / \mathrm{hr}$ & $2.7 \mathrm{u} / \mathrm{hr}$ & $52.23 \mathrm{u} / \mathrm{hr}$ & $66.16 \mathrm{u} / \mathrm{hr}$ & $82.45 \%$ \\
\hline
\end{tabular}

deberg's central limit theorem holds.

In a further analysis, the results from both methodologies also show some interesting conclusions. As it was expected, the conventional methodology underestimates the variability of the manufacturing process, so it tends to loose the tolerances allocated in the optimization problem resulting in a lower manufacturing cost. It expects a quality loss cost of $10.6 \mathrm{u} / \mathrm{hr}$, however this result is misleading since it overlooks other sources of variation, such as the thermal spindle expansion or the cuttingtool wear effect on part dimensions. Thus, the conventionl methodology presents a locators design with lower precision (wider initial tolerances -see Fig. 7-) with a lower frequency of maintenance actions (larger operation times between fixture maintenance actions -see Fig. 8-) than the proposed methodology. Another important difference of both methodologies is presented in the cutting-tool replacement cost and thus, in the admissible cutting-tool wear values. The results show that the conventional methodology replaces the cutting-tools when the maximum tool wear is reached $(0.4 \mathrm{~mm}$ of tool-wear according to vendor's recommendations) since this methodology only takes into account the admissible tool wear in the cost term of the optimization function (under-use cutting-tool cost and replacement cost) and overlooks its effect on dimensional part quality. Instead, the proposed methodology restricts the admissible tool wear $(0.060,0.057$ and $0.057 \mathrm{~mm}$ in the first, second and third station respectively, see Fig. 9) to ensure part quality but the cutting-tool replacement cost is increased.

\section{CONCLUSIONS AND FUTURE WORK}

In this paper, a process-oriented tolerancing methodology has been developed to incorporate additional process variables, cost functions and quality constraints that have not been included yet in existing research. In order to add these additional elements, an extended SoV model was applied. Through this process-oriented approach, process variables such as cutting-tool wear and machinetool thermal state, and fixture maintenance and cutting-tool replacement costs can be integrated, and the resolution of the optimization problem through stochastic optimization algorithms such as GA and MADS can lead to achieve a real minimum manufacturing cost. A case study was conducted to demonstrate the potentials of the proposed process-oriented tolerancing methodology. The results revealed that, without including KCCs related to thermal state of the spindles or the allowable cutting-tool wear, the tolerance allocation decisions may be misleading, increasing both the number of non-conforming parts and the final manufacturing cost.

This research work shows the potential benefits of using the extended SoV model for tolerance allocation improving the rate of parts within specifications and the manufacturing cost. As future work, the authors suggest two directions of research: 

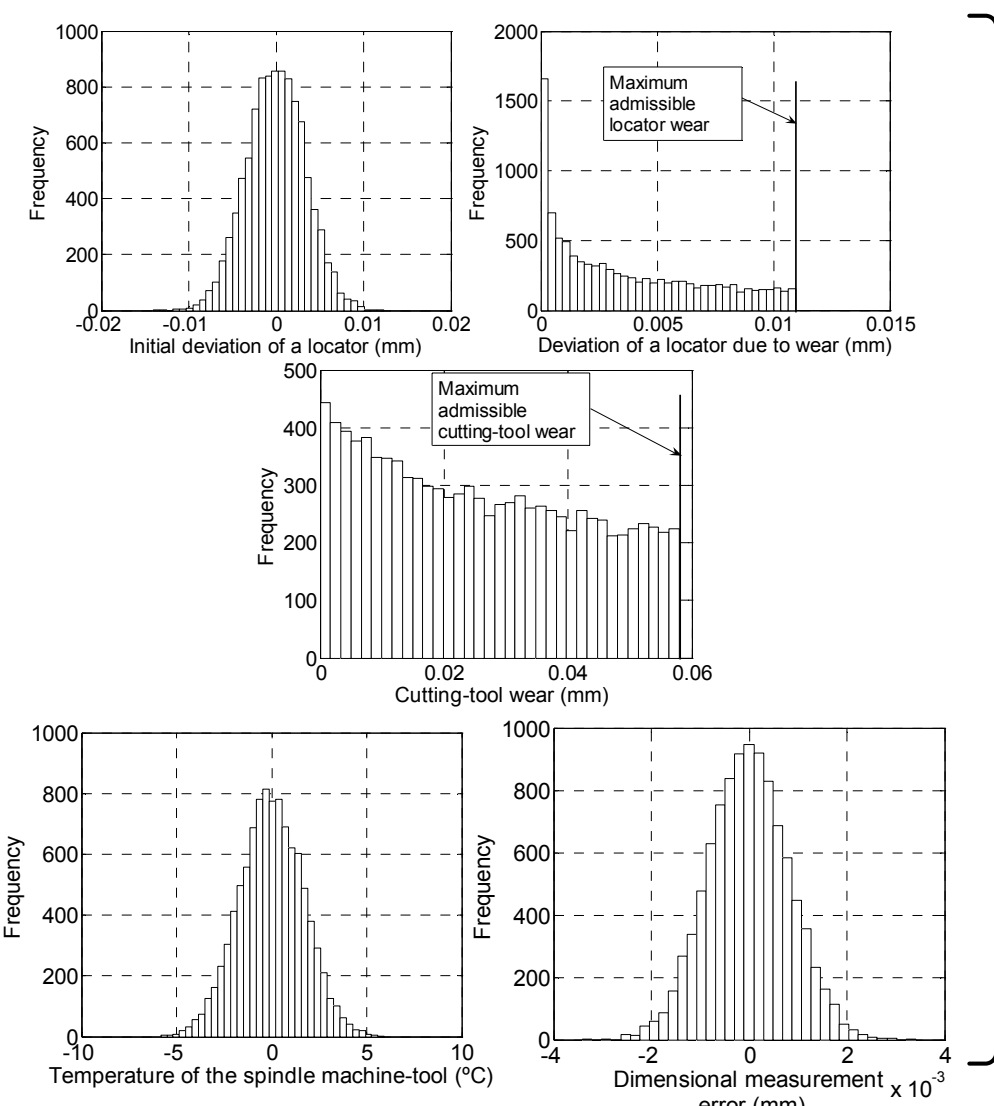

error $(\mathrm{mm})$

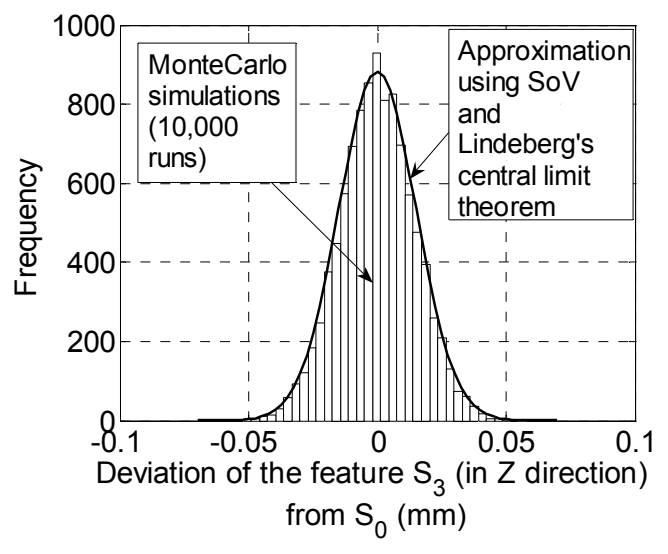

Figure 6: Pdf of some of the KCCs for the case study and the resulting pdf of the deviation of feature $S_{3}$.

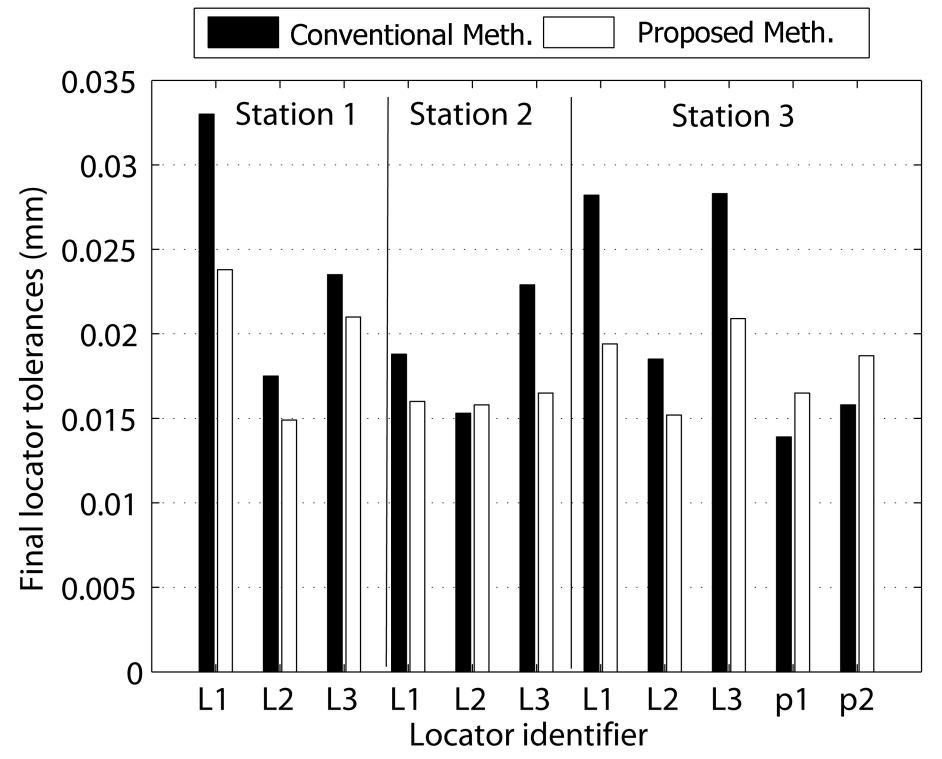

Figure 7: Design tolerances for fixture locators. 


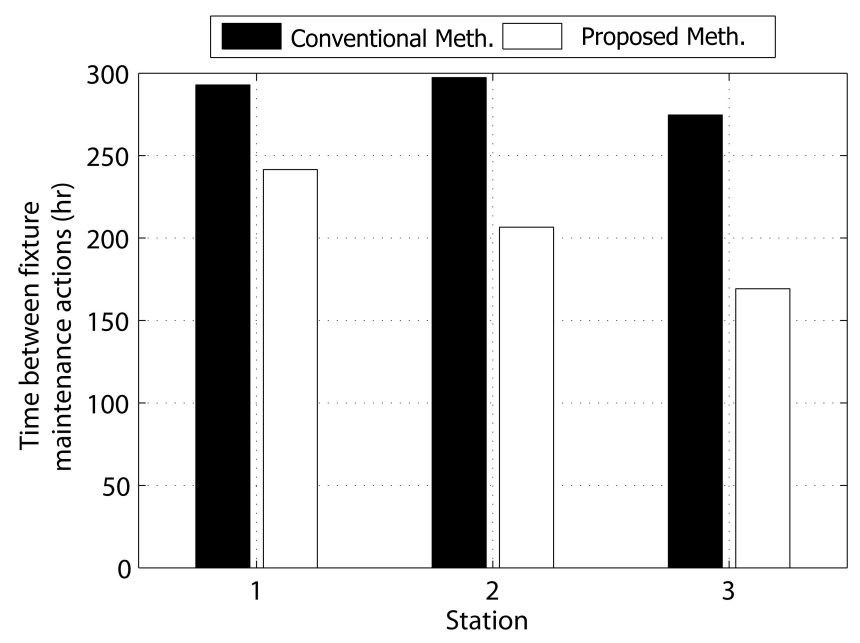

Figure 8: Time between two consecutive fixture maintenance actions (in hours) at each machining station.

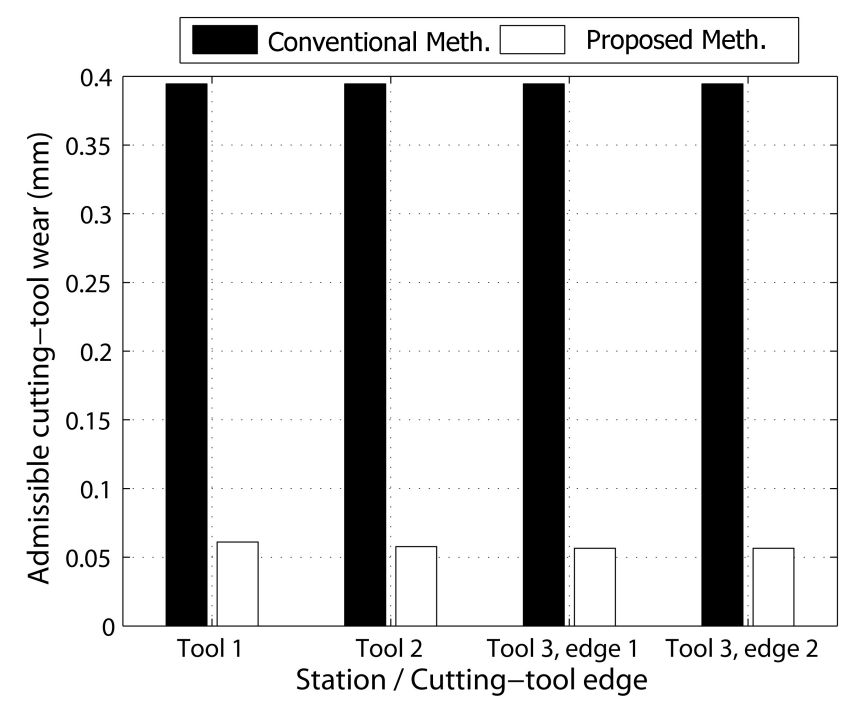

Figure 9: Admissible cutting-tool wear at each cutting-tool edge before a cutting-tool replacement is conducted. 
- Increase the comprehensiveness of the extended SoV model in order to consider new variables that can have an impact of costs or part quality. For instance, it could interesting to analyze how cutting conditions (cutting speed, feed rate, etc.) can increase the productivity but, at the same time, decrease the part quality due to deflexions or excesive cutting-tool wear rate. Therefore, the process-oriented toleranced problem will also take into account cutting conditions through the extended SoV model.

- Modify the proposed methodology in order to cover other GD\&T specifications and other nonpunctual fixtures more common in industry such as jaws or similar.

\section{ACKNOWLEDGEMENTS}

This work has been partially supported by Fundació Caixa-Castelló Bancaixa (Research Promotion 2007, 2009 and 2011). The authors greatly acknowledge the investigation support by the NSF Engineering Research Center for Reconfigurable Manufacturing System at University of Michigan.

\section{References}

[1] Y. Hong, T. Chang, A comprehensive review of tolerancing research, International Journal of Production Research 40 (11) (2002) 2425-2459.

[2] P. Drake, Dimensioning and tolerancing handbook, McGraw-Hill, 1999.

[3] Y. Ding, J. Jin, D. Ceglarek, J. Shi, Process-oriented tolerancing for multi-station assembly systems, IIE Transactions 37 (2005) 493-508.

[4] J. Shi, Stream of Variation Modeling and Analysis for Multistage, CRC Press Taylor and Francis Group, 2007.

[5] S. Du, L. Xi, J. Ni, P. Ershun, C. R. Liu, Product lifecycle-oriented quality and productivity improvement based on stream of variation methodology, Computers in Industry 59 (2 - 3) (2008) $180-192$.

[6] Y. Ding, J. J. Shi, D. Ceglarek, Diagnosability analysis of multi-station manufacturing processes, Journal of Dynamic Systems Measurement and Control-Transactions of the ASME 124 (1) (2002) 1-13.

[7] A. Khan, D. Ceglarek, Sensor optimization for fault diagnosis in multi-fixture assembly systems with distributed sensing, ASME Transactions, Journal of Manufacturing Science and Engineering 122 (2000) 215.

[8] Q. Liu, Y. Ding, Y. Chen, Optimal coordinate sensor placements for estimating mean and variance components of variation sources, IIE Transactions 37 (9) (2005) 877-889.

[9] S. Zhou, Q. Huang, J. Shi, State space modeling of dimensional variation propagation in multistage machining process using differential motion vectors, IEEE Transactions on Robotics and Automation 19 (2) (2003) 296-309.

[10] J. V. Abellan-Nebot, J. Liu, F. R. Subiron, Design of multi-station manufacturing processes by integrating the stream-of-variation model and shop-floor data, Journal of Manufacturing Systems 30 (2) (2011) $70-82$. 
[11] J. V. Abellan-Nebot, J. Liu, F. R. Subiron, J. Shi, State space modeling of variation propagation in multistation machining processes considering machining-induced variations, Journal of Manufacturing Science and Engineering, Transactions of the ASME 134 (2).

[12] D. Djurdjanovic, J. Ni, Online stochastic control of dimensional quality in multistation manufacturing systems, Proceedings of the Institution of Mechanical Engineers Part B-Journal of Engineering Manufacture 221 (5) (2007) 865-880.

[13] J. V. Abellan-Nebot, J. Liu, F. R. Subiron, Quality prediction and compensation in multi-station machining processes using sensor-based fixtures, Robotics and Computer-Integrated Manufacturing 28 (2) (2012) $208-219$.

[14] Y. Chen, Y. Ding, J. Jin, D. Ceglarek, Integration of process-oriented tolerancing and maintenance planning in design of multistation manufacturing processes, IEEE Transactions on Automation Science and Engineering 3 (4) (2006) 440-453.

[15] Q. Huang, J. Shi, Simultaneous tolerance synthesis through variation propagation modeling of multistage manufacturing processes, NAMRI/SME Transactions 31 (2003) 515-522.

[16] J. Liu, J. Shi, S. J. Hu, Quality-assured setup planning based on the stream-of-variation model for multi-stage machining processes, IIE Transactions 41 (12) (2009) 323-334.

[17] R. Ramesh, M. A. Mannan, A. N. Poo, Error compensation in machine tools - a review part I: geometric, cutting-force induced and fixture-dependent errors, International Journal of Machine Tools and Manufacture 40 (9) (2000) 1235-1256.

[18] J. V. Abellan-Nebot, J. Liu, F. Romero, Limitations of the current state space modelling approach in multistage machining processes due to operation variations, AIP Conference Proceedings 1181 (1) (2009) 231-243. doi:10.1063/1.3273636.

[19] R. Mantripragada, D. E. Whitney, The datum flow chain: A systematic approach to assembly design and modeling, Research in Engineering Design-Theory Applications and Concurent Engineering 10 (1998) 150-156.

[20] J. Jin, J. Shi, State space modeling of sheet metal assembly for dimensional control, ASME Transactions, Journal of Manufacturing Science and Engineering 121 (1999) 756-762.

[21] W. Huang, J. Lin, Z. Kong, Stream-of-variation (SOVA) modeling II: A generic 3D variation model for rigid body assembly in multi-station assembly processes, Transaction of ASME, Journal of Manufacturing Science and Engineering 129 (2007) 832-842.

[22] D. Djurdjanovic, J. Ni, Dimensional errors of fixtures, locating and measurement datum features in the stream of variation modeling in machining, Journal of Manufacturing Science and Engineering-Transactions of the ASME 125 (4) (2003) 716-730.

[23] X.-J. Wan, C.-H. Xiong, X.-F. Wang, X.-M. Zhang, Y.-L. Xiong, Analysis-synthesis of dimensional deviation of the machining feature for discrete-part manufacturing processes, International Journal of Machine Tools and Manufacture 49 (15) (2009) 1214 - 1233.

[24] A. Wirtz, Vectorial tolerancing for quality control and functional analysis in design, in: CIRP Internation Working Seminar on Computer Aided Tolerancing, Penn State University, 1991. 
[25] C.-C. Wu, Z. Chen, G.-R. Tang, Component tolerance design for minimum quality loss and manufacturing cost, Computers in Industry 35 (3) (1998) 223 - 232.

[26] P. Joshi, Jigs and Fixtures Design Manual, McGraw-Hill, 2003.

[27] H. P. Bloch, F. K. Geitner, Machinery Component Maintenance and Repair, Gulf Professional Publishing, 2003.

[28] ASM-Handbook, ASM Metals Handbook, 9th Edition, Vol. 16 Machining, 2003.

[29] G. Taguchi, S. Chowdhury, Y. Wu, Taguchi's Quality Engineering Handbook, John Wiley and Sons Ltd, 2004.

[30] S. Zhang, Z. Sha, R. Kang, A physical machining accuracy predicting model in turning, Key Engineering Materials 329 (2007) 675-680.

[31] J. P. Loose, Q. Zhou, S. Y. Zhou, D. Ceglarek, Integrating GD\&T into dimensional variation models for multistage machining processes, International Journal of Production Research 48 (11) (2010) 3129-3149.

[32] F. M. Dekking, C. Kraaikamp, H. P. Lopuha, L. E. Meester, A Modern Introduction to Probability and Statistics: Understanding Why and How, Springer, 2005.

[33] P. Billingsley, Probability and measure, 3rd Edition, John Wiley \& Sons Inc., New York, 1995.

[34] P. K. Singh, S. C. Jain, P. K. Jain, Advanced optimal tolerance design of mechanical assemblies with interrelated dimension chains and process precision limits, Computers in Industry 56 (2) (2005) $179-194$.

[35] The Mathworks, Matlab Optimization Toolbox User's Guide, Available via http://www.mathworks.com/help/toolbox/gads/. Accessed 15 March 2011 (2011).

\section{Appendix A: probability density functions of process variables}

Fixture wear is assumed to follow a quadratic curve with operation time expressed by the equation

$$
w_{k}^{l}=G_{k} \cdot\left(t_{k}^{o p}\right)^{2}
$$

where $w_{k}^{l}$ is the locator wear at the operation time $t_{k}^{o p}$ (note that if $t_{k}^{o p}=t_{k}^{a}$, then the locator wear is the admissible maximum wear and thus, $\left.w_{k}^{l}=\Delta l_{k}^{w}\right)$, and $t_{k}^{o p} \in\left[0, t_{k}^{a}\right]$. As the part inspection is assumed to be conducted uniformly along the production when a large number of parts are inspected, the pdf of the variable $t_{k}^{o p}$ can be considered as a uniform random distribution in the range $\left[0, t_{k}^{a}\right]$. Thus, the pdf of fixture wear in the $k$ station can be obtained by the change of variable formula [32]. By this formula, if $y=r(x)$ where $r$ is differentiable and $f_{X}(x)$ is the pdf of $x$, then the pdf of $y$ is calculated as:

$$
g(y)=f_{X}\left(r^{-1}(y)\right) \cdot\left|\frac{d r^{-1}(y)}{d y}\right|
$$


Applying this formula, the pdf of fixture wear is

$$
g\left(w_{k}^{l}\right)=\frac{1}{t_{k}^{a}} \cdot\left|\frac{1}{\sqrt{4 G_{k} \cdot w_{k}^{l}}}\right|,
$$

where $w_{k}^{l} \in\left[0, \Delta l_{k}^{w}\right]$ and $\Delta l_{k}^{w}=G_{k} \cdot\left(t_{k}^{a}\right)^{2}$. For the numerical values applied in the case study (Table $4)$, the mean and variance value of this process variable can be obtained as:

$$
\begin{aligned}
E\left(w_{k}^{l}\right) & =0.169 \cdot 10^{-9} \cdot\left(t_{k}^{a}\right)^{3}, \\
\operatorname{Var}\left(w_{k}^{l}\right) & =0.175 \cdot 10^{-24} \cdot\left(t_{k}^{a}\right)^{5}\left(429 \cdot\left(t_{k}^{a}\right)^{2}-326000 \cdot t_{k}^{a}+0.112 \cdot 10^{9}\right) .
\end{aligned}
$$

The cutting-tool wear variable is assumed to follow a quadratic curve with the machining time in the form:

$$
w_{\tau, k}^{\varphi}=E_{\tau, k}^{\varphi} \cdot t_{\tau, k}^{m}+F_{\tau, k}^{\varphi} \cdot\left(t_{\tau, k}^{m}\right)^{2}
$$

where $w_{\tau, k}^{\varphi}$ is the cutting-tool wear at the machining time $t_{\tau, k}^{m}$, and $t_{\tau, k}^{m} \in\left[0, t_{\tau, k}^{b_{\varphi}}\right]$. Following the same procedure as shown above, the pdf of the cutting-tool wear variable is defined as:

$$
h\left(w_{\tau, k}^{\varphi}\right)=\frac{1}{t_{\tau, k}^{b_{\varphi}}} \cdot\left|\frac{1}{\sqrt{\left(E_{\tau, k}^{\varphi}\right)^{2}+4 \cdot F_{\tau, k}^{\varphi} \cdot w_{\tau, k}^{\varphi}}}\right|,
$$

where $w_{\tau, k}^{\varphi} \in\left[0, V_{\tau, k}^{\varphi}\right]$ and $V_{\tau, k}^{\varphi}=E_{\tau, k}^{\varphi} \cdot t_{\tau, k}^{b_{\varphi}}+F_{\tau, k}^{\varphi} \cdot\left(t_{\tau, k}^{b_{\varphi}}\right)^{2}$. For the numerical case study, the mean and variance value of this process variable are defined as follows:

$$
\begin{aligned}
E\left(w_{\tau, k}^{\varphi}\right)= & 0.00322+\left(\sqrt{12800+8590 t_{\tau, k}^{b \varphi}+1440\left(t_{\tau, k}^{b \varphi}\right)^{2}}\right)\left(2.85 \cdot 10^{-5}+0.959 \cdot 10^{-5} t_{\tau, k}^{b \varphi}+0.161 \cdot 10^{-5}\left(t_{\tau, k}^{b \varphi}\right)^{2}\right), \\
\operatorname{Var}\left(w_{\tau, k}^{\varphi}\right)= & \left(\sqrt{12800+8590 t_{\tau, k}^{b \varphi}+1440\left(t_{\tau, k}^{b}\right)^{2}}\right)\left(0.857 \cdot 10^{-6}-0.271 \cdot 10^{-6} t_{\tau, k}^{b \varphi}+0.105 \cdot 10-7\left(t_{\tau, k}^{b \varphi}\right)^{2}+0.209 \cdot 10^{-7}\left(t_{\tau, k}^{b \varphi}\right)^{3}\right. \\
& \left.+0.260 \cdot 10^{-8}\left(t_{\tau, k}^{b \varphi}\right)^{4}+0.171 \cdot 10^{-9}\left(t_{\tau, k}^{b}\right)^{5}+0.955 \cdot 10^{-11}\left(t_{\tau, k}^{b \varphi}\right)^{6}+0.857 \cdot 10^{-6}\right)-9.69 \cdot 10^{-5}-0.196 \cdot 10^{-5} t_{\tau, k}^{b \varphi} \\
& +0.910 \cdot 10^{-5}\left(t_{\tau, k}^{b \varphi}\right)^{2}+0.135 \cdot 10^{-5}\left(t_{\tau, k}^{b \varphi}\right)^{3}-0.647 \cdot 10^{-6}\left(t_{\tau, k}^{b \varphi}\right)^{4}-0.153 \cdot 10^{-6}\left(t_{\tau, k}^{b \varphi}\right)^{5}-0.858 \cdot 10^{-8}\left(t_{\tau, k}^{b \varphi}\right)^{6} \quad \text { (A.9) }
\end{aligned}
$$

\section{Appendix B: Derivation of matrix $\mathbf{C}_{P_{p}}$ for direct measurements}

The dimensional deviation of features inspected by a direct measurement can be estimated by Eq. (3) (and their variance with Eq. (17)) if one can estimate the deviations of process variables. However, this equation expresses the deviation of the LCS of the feature inspected w.r.t the GD\&T reference datum, and we are interested in evaluating the deviation of all boundary points of the feature inspected in order to analyse if the feature is within the tolerance specification. Thus, we want to evaluate the boundary point deviations w.r.t the GD\&T reference datum knowing the LCS deviation of the inspected feature. This relationship can be known applying the Corollary 1 presented in [9]. This Corollary is defined as follows. Consider two features 1 and 2 and a global coordinate system R. Given the deviation of the feature 1 w.r.t. $\mathrm{R}, \mathbf{x}_{1}^{R}$, and the deviation of feature 2 w.r.t. feature $1, \mathbf{x}_{2}^{1}$, then: 


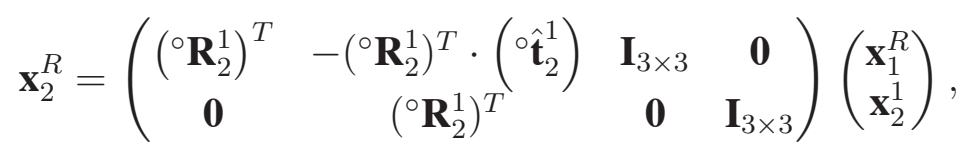

where ${ }^{\circ} \mathbf{R}_{2}^{1}$ is the nominal rotational matrix between feature 1 and $2 ;{ }^{\circ} \hat{\mathbf{t}}_{2}^{1}$ is the skew symmetric matrix obtained from the nominal location vector ${ }^{\circ} \mathbf{t}_{2}^{1}=\left[t_{2 x}^{1}, t_{2 y}^{1}, t_{2 z}^{1}\right]^{T}$ and it is defined as ${ }^{\circ} \hat{\mathbf{t}}_{2}^{1}=\left[\left[0,-t_{2 z}^{1}, t_{2 y}^{1}\right]\right.$; $\left.\left[t_{2 z}^{1}, 0,-t_{2 x}^{1}\right] ;\left[-t_{2 y}^{1}, t_{2 x}^{1}, 0\right]\right]$; and $\mathbf{I}_{3 \times 3}$ is a $3 \times 3$ identity matrix.

Applying this corollary and assuming rigid parts, the deviation of the boundary points defined in step 2 w.r.t. the GD\&T reference datum $\left(\mathbf{x}_{P_{p}^{S_{q}}}^{S_{m}}\right)$ is related to the deviation of the LCS attached to the controlled feature w.r.t. the GD\&T reference datum $\left(\mathbf{x}_{S_{q}}^{S_{m}}\right)$ by the matrix $\mathbf{C}_{P_{p}}$ according to the following equation:

$$
\mathbf{x}_{P_{p}^{S_{q}}}^{S_{m}}=\mathbf{C}_{P_{p}} \cdot \mathbf{x}_{S_{q}}^{S_{m}}, \quad \forall P_{p} \in S_{q},
$$

where

$$
\mathbf{C}_{P_{p}}=\left(\begin{array}{cc}
\mathbf{I}_{3 \times 3} & -\left({ }^{\circ} \hat{\mathbf{t}}_{P_{p}^{S_{q}}}^{S_{q}}\right) \\
\mathbf{0} & \mathbf{I}_{3 \times 3}
\end{array}\right) .
$$

This result is obtained straight forward from Corollary 1 since by assuming rigid parts, the DMV $\mathbf{x}_{P_{p}^{S}}^{S_{q}}$ is always zero.

\section{Appendix C: Derivation of matrix $\mathrm{K}$ for indirect measurements}

For indirect measurements, a controlled feature and a GD\&T reference datum feature are measured in the inspection station w.r.t the measurement datum. Thus, Eq. (3) gives us the deviation of the LCS of both features w.r.t the measurement datum. However, we are interested in the deviation of the boundary points of the controlled feature w.r.t the GD\&T reference datum feature. Thus, we have to express the deviation of the LCS of the controlled feature w.r.t the LCS of the GD\&T reference datum feature. For this purpose, the Corollary 2 presented in [9] can be applied. This Corollary is defined as follows. Consider feature 1 and feature 2 and a global coordinate system R. Given the deviation of feature 1 w.r.t. $\mathrm{R}, \mathbf{x}_{1}^{R}$, and the deviation of feature 2 w.r.t. $\mathrm{R}, \mathbf{x}_{2}^{R}$, then:

$$
\mathbf{x}_{2}^{1}=\left(\begin{array}{cccc}
-\left({ }^{\circ} \mathbf{R}_{2}^{1}\right)^{T} & \left({ }^{\circ} \mathbf{R}_{2}^{1}\right)^{T} \cdot\left({ }^{\circ} \hat{\mathbf{t}}_{2}^{1}\right) & \mathbf{I}_{3 \times 3} & \mathbf{0} \\
\mathbf{0} & -\left({ }^{\circ} \mathbf{R}_{2}^{1}\right)^{T} & \mathbf{0} & \mathbf{I}_{3 \times 3}
\end{array}\right)\left(\begin{array}{c}
\mathbf{x}_{1}^{R} \\
\mathbf{x}_{2}^{R}
\end{array}\right) .
$$

Applying this corollary, the DMV of the controlled feature w.r.t the GD\&T reference datum $\left(\mathbf{x}_{S_{q}}^{S_{m}}\right)$ is related to the DMVs of the GD\&T reference datum and the controlled feature w.r.t. the measurement datum feature ( $\mathbf{x}_{S_{m}}^{S_{D}}$ and $\mathbf{x}_{S_{q}}^{S_{D}}$ respectively) by the matrix $\mathbf{K}$ as follows:

$$
\mathbf{x}_{S_{q}}^{S_{m}}=\mathbf{K} \cdot\left[\begin{array}{ll}
\mathbf{x}_{S_{m}}^{S_{D}} & \mathbf{x}_{S_{q}}^{S_{D}}
\end{array}\right]^{T},
$$

where

$$
\mathbf{K}=\left(\begin{array}{cccc}
-\left({ }^{\circ} \mathbf{R}_{S_{q}}^{S_{m}}\right)^{T} & \left({ }^{\circ} \mathbf{R}_{S_{q}}^{S_{m}}\right)^{T} \cdot\left({ }^{\circ} \hat{\mathbf{t}}_{S_{q}}^{S_{m}}\right) & \mathbf{I}_{3 \times 3} & \mathbf{0} \\
\mathbf{0} & -\left({ }^{\circ} \mathbf{R}_{S_{q}}^{S_{m}}\right)^{T} & \mathbf{0} & \mathbf{I}_{3 \times 3}
\end{array}\right) .
$$

